\title{
OPTIMAL CONTROL OF THE UNDAMPED LINEAR WAVE EQUATION WITH MEASURE VALUED CONTROLS*
}

\author{
KARL KUNISCH $^{\dagger}$, PHILIP TRAUTMANN ${ }^{\dagger}$, AND BORIS VEXLER ${ }^{\ddagger}$
}

\begin{abstract}
Measure valued optimal control problems governed by the linear wave equation are analyzed. The space of vector measures $\mathcal{M}\left(\Omega_{c}, L^{2}(I)\right)$ is chosen as control space and the corresponding total variation norm as the control cost functional. The support of the controls (sparsity pattern) is time-independent, which is desired in many applications, e.g., inverse problems or optimal actuator placement. New regularity results for the linear wave equation are proven and used to show the well-posedness of the control problem in all three space dimensions. Furthermore first order optimality conditions are derived and structural properties of the optimal control are investigated. Higher regularity of optimal controls in time is shown on the basis of the regularity results for the state. Finally the optimal control problem is used to solve an inverse source problem.
\end{abstract}

Key words. optimal control, sparsity, wave equation, regularity, inverse problem

AMS subject classifications. 35L05, 49J52, 49K20, 49M15, 90C48

DOI. $10.1137 / 141001366$

1. Introduction. This work is dedicated to optimal control problems of the following form:

$$
\min _{u, y} J(y)+\alpha\|u\|_{\mathcal{M}\left(\Omega_{c}, L^{2}(I)\right)}
$$

subject to

$$
\left\{\begin{aligned}
\partial_{t t} y-c^{2} \Delta y & =u & & \text { in } I \times \Omega, \\
y & =0 & & \text { on } I \times \partial \Omega, \\
\left(y, \partial_{t} y\right) & =\left(y_{0}, y_{1}\right) & & \text { in }\{0\} \times \Omega .
\end{aligned}\right.
$$

The set $\Omega \subset \mathbb{R}^{d}, d \in\{1,2,3\}$, denotes a bounded domain with a sufficiently smooth boundary $\partial \Omega$ and $I=(0, T), T>0$, is the time interval. Furthermore the control set $\Omega_{c}$ is a compact subset of $\Omega$. Problem $(\mathcal{P})$ constitutes an optimal control problem governed by the linear undamped wave equation with constant wave speed $c \in \mathbb{R}_{+}^{*}$ and homogeneous Dirichlet boundary conditions. Equation (1.1) describes the generation of acoustic waves by the source $u$ and its evolution in a homogeneous medium. More complicated (vector-valued) versions of this equation model the behavior of seismic waves. The control $u$ is chosen from $\mathcal{M}\left(\Omega_{c}, L^{2}(I)\right)$, the space of finite vector measures with values in $L^{2}(I)$, and enters the state equation as a source term. The cost functional $J$ consists of a quadratic tracking functional and a control cost functional. We will concentrate on a tracking functional of the form

$$
J(y)=\frac{1}{2}\left\{\nu_{1}\left\|y-z_{1}\right\|_{L^{2}(I \times \Omega)}^{2}+\nu_{2}\left\|y(T)-z_{2}\right\|_{L^{2}(\Omega)}^{2}+\nu_{3}\left\|\partial_{t} y(T)-z_{3}\right\|_{H^{-1}(\Omega)}^{2}\right\}
$$

\footnotetext{
* Received by the editors December 23, 2014; accepted for publication (in revised form) February 5, 2016; published electronically May 12, 2016.

http://www.siam.org/journals/sicon/54-3/100136.html

$\dagger$ Institute for Mathematics and Scientific Computing, University of Graz, Heinrichstrasse 36, A-8010 Graz, Austria (karl.kunisch@uni-graz.at, philip.trautmann@uni-graz.at). The second author received support from the International Research Training Group IGDK1754, funded by the DFG and FWF.

${ }^{\ddagger}$ Chair of Optimal Control, Technische Universität München, Boltzmannstraße 3, 85748 Garching bei München, Germany (vexler@ma.tum.de).
} 
with $\nu_{i} \geq 0$, so $\mathcal{C}\left(\bar{I}, L^{2}(\Omega)\right) \cap \mathcal{C}^{1}\left(\bar{I}, H^{-1}(\Omega)\right)$ regularity of the state $y$ will be essential. The nonsmooth control cost term is given by the total variation norm for vector measures $u \in \mathcal{M}\left(\Omega_{c}, L^{2}(I)\right)$ and is weighted by a control cost parameter $\alpha>0$.

The choice of the control space $\mathcal{M}\left(\Omega_{c}, L^{2}(I)\right)$ can be motivated by the following considerations. Every vector measure $u \in \mathcal{M}\left(\Omega_{c}, L^{2}(I)\right)$ can be decomposed into a space-dependent measure part $|u| \in \mathcal{M}\left(\Omega_{c}\right)^{+}$and a space-time-dependent function $u^{\prime}(x, t) \in L^{1}\left((\Omega,|u|), L^{2}(I)\right)$ (Radon-Nikodym derivative); in particular it has the form

$$
\mathrm{d} u(t)=u^{\prime}(x, t) \mathrm{d}|u| .
$$

Therefore the support of a vector measure $u \in \mathcal{M}\left(\Omega_{c}, L^{2}(I)\right)$ (sparsity pattern) is time-independent and thus the control space $\mathcal{M}\left(\Omega_{c}, L^{2}(I)\right)$ can be seen as a measurevalued generalization of group (joint, directional) sparsity. This concept is well known in the context of compressed sensing, e.g., [16]. We note that pointwise controls with fixed positions and time-dependent intensities

$$
u(t)=\sum_{i=1}^{N} u_{i}(t) \delta_{x_{i}},\left\{u_{i}\right\}_{i=1, \ldots, N} \subset L^{2}(I),\left\{x_{i}\right\}_{i=1, \ldots, N} \subset \Omega_{c},
$$

or more general controls of the form

$$
u(t)=\sum_{i=1}^{N} u_{i}(t) \mu(x), \mu \in \mathcal{M}\left(\Omega_{c}\right),
$$

e.g., $\mu$ as a line or surface measure, can be realized within $\mathcal{M}\left(\Omega_{c}, L^{2}(I)\right)$. Such controls are of great interest in the context of inverse problems or optimal actuator placement problems. In particular point sources of the form (1.3) are often used as simple models for localized acoustic or seismic events, e.g., explosions, earthquakes or volcano eruptions. The optimal control problem which uses directly the ansatz (1.3) and therefore optimizes the position of the point sources and their intensities directly is nonconvex, whereas the formulation in the space of vector measures is a convex optimization problem which also optimizes for the locations of the potential point sources and their intensities. But it can be guaranteed only under certain assumptions on the optimal adjoint state that the optimal control of problem $(\mathcal{P})$ has the structure (1.3). These conditions will emerge from the first order optimality conditions of $(\mathcal{P})$.

The particular control space was first investigated in a paper [20], which deals with optimal control problems governed by linear parabolic PDEs. The authors establish the well-posedness of the optimal control problem and derive first order optimality conditions. We will rely partly on their analysis. In [18] the concept of group sparsity was introduced to optimal control of PDEs. The functional $\|u\|_{L^{1}\left(\Omega_{c}, L^{2}(I)\right)}+$ $\|u\|_{L^{2}\left(I \times \Omega_{c}\right)}^{2}$ is used as control cost term. In [7] the authors consider the control space $L^{2}\left(I, \mathcal{M}\left(\Omega_{c}\right)\right)$, the space of $L^{2}$-functions in time with values in $\mathcal{M}\left(\Omega_{c}\right)$, in connection with parabolic optimal control problems. The major difference between our control space and their control space is that the latter allows for a time-dependent support of the measure (sparsity pattern), e.g., moving point sources are allowed as controls. The article [10] studies controls from $\mathcal{M}\left([0, \hat{T}] \times \Omega_{c}\right)$ with $\hat{T}<T$, therefore space-time Dirac measures are allowed as controls. Furthermore it can be guaranteed that the optimal controls consist of a finite sum of Dirac measures in the setting of [10]. In [9] the authors investigate a measure valued optimal control problem involving the 
initial data as control. Measure valued controls were also investigated in connection with elliptic PDEs; see, e.g., [11, 12] and semilinear elliptic PDEs [8]. In [4] measure valued controls are considered from the inverse problem point of view.

The main contributions of the present paper are the following. First we show improved regularity results for (1.1) with controls from $\mathcal{M}\left(\Omega_{c}, L^{2}(I)\right)$. In particular we prove

$$
y \in \mathcal{C}\left(\bar{I},\left[H^{2}(\Omega) \cap H_{0}^{1}(\Omega), L^{2}(\Omega)\right]_{1 / 2-\theta_{d}}\right) \cap \mathcal{C}^{1}\left(\bar{I},\left[L^{2}(\Omega),\left(H^{2}(\Omega) \cap H_{0}^{1}(\Omega)\right)^{*}\right]_{-\theta_{d}}\right)
$$

with $\theta_{d}=(1-d) / 4$. This is a $\frac{1}{2}+\varepsilon$ improvement in Sobolev regularity over standard regularity theory for the linear wave equation, which uses the embedding

$$
\mathcal{M}\left(\Omega_{c}, L^{2}(I)\right) \hookrightarrow L^{2}\left(I, \mathcal{M}\left(\Omega_{c}\right)\right) \hookrightarrow L^{2}\left(I, H^{-d / 2-\varepsilon}(\Omega)\right)
$$

for arbitrary $\varepsilon>0$, e.g., [26, 23]. The proof is based on improved regularity results for (1.1) with $u(t)=f(t) \delta_{\tilde{x}} \in \mathcal{M}\left(\Omega_{c}, L^{2}(I)\right), f \in L^{2}(I)$, and $\tilde{x} \in \Omega_{c}$ which can be found in $[25,28,24]$. Furthermore the proof uses explicitly the properties of the space $\mathcal{M}\left(\Omega_{c}, L^{2}(I)\right)$ and is not based on the embedding $\mathcal{M}\left(\Omega_{c}, L^{2}(I)\right) \hookrightarrow L^{2}\left(I, \mathcal{M}\left(\Omega_{c}\right)\right)$. The second important result of this paper is the well-posedness of problem $(\mathcal{P})$. The proof utilizes the mentioned improved regularity results for the state variable. Furthermore improved regularity of the optimal control in time is established, namely, $\bar{u} \in \mathcal{C}^{2-d, \frac{1}{2}-\varepsilon}\left(\bar{I}, \mathcal{M}\left(\Omega_{c}\right)\right)$ for $d=1,2$ and any $0<\varepsilon \leq 1 / 2$. Moreover we adopt the problem formulation $(\mathcal{P})$ for the solution of an inverse problem motivated by a geophysical application, namely, the reconstruction of the locations and intensities of seismic events from noisy observations of the emitted waves.

The outline of this paper is as follows. In section 2 the space $\mathcal{M}\left(\Omega_{c}, L^{2}(I)\right)$ is introduced. In section 3 the required results from interpolation theory of Sobolev spaces are collected. In section 4 well-posedness of the wave equation and dual wave equation for different regularity classes of data is discussed. Section 5 is dedicated to improved regularity for the primal and dual wave equation. In section 6 well-posedness of the control problem $(\mathcal{P})$ is proven. Section 7 is concerned with the derivation of first order optimality conditions for problem $(\mathcal{P})$. In section 8 the discretization of $(\mathcal{P})$ with finite elements and its algorithmic solution by a continuation method are discussed. Finally in section 9 the problem formulation $(\mathcal{P})$ is applied to an inverse source problem.

2. The space $\mathcal{M}\left(\Omega_{c}, L^{2}(I)\right)$. In this section we introduce the control space $\mathcal{M}\left(\Omega_{c}, L^{2}(I)\right)$ and its properties. Let $\mu: \mathcal{B}\left(\Omega_{c}\right) \rightarrow L^{2}(I)$ be a countably additive mapping on the Borel sets $\mathcal{B}\left(\Omega_{c}\right)$ of $\Omega_{c}$ with values in $L^{2}(I)$. For $\mu$ we denote by $|\mu| \in \mathcal{M}^{+}\left(\Omega_{c}\right)$ (positive regular Borel measure) the total variation measure defined by

$$
|\mu|(B)=\sup _{\pi} \sum_{E \in \pi}\|\mu(E)\|_{L^{2}(I)},
$$

where $\pi$ is the set of all disjoint partitions of $B \in \mathcal{B}\left(\Omega_{c}\right)$. The space

$$
\mathcal{M}\left(\Omega_{c}, L^{2}(I)\right)=\left\{\mu: \mathcal{B}\left(\Omega_{c}\right) \rightarrow L^{2}(I): \quad \mu \text { countably additive, }|\mu|\left(\Omega_{c}\right)<\infty\right\}
$$

is the space of vector measures with values in $L^{2}(I)$. Equipped with the norm

$$
\|\mu\|_{\mathcal{M}\left(\Omega_{c}, L^{2}(I)\right)}=|\mu|\left(\Omega_{c}\right)
$$


it is a Banach space. The support of $\mu$, respectively, of its total variation measure $|\mu|$, is defined by

$$
\operatorname{supp} \mu=\operatorname{supp}|\mu|=\Omega_{c} \backslash\left(\bigcup\left\{B \text { open in } \Omega_{c}|| \mu \mid(B)=0\right\}\right) .
$$

The vector measure $\mu$ possesses a Radon-Nikodym derivative (see [21]),

$$
\mu^{\prime} \in L^{\infty}\left(\left(\Omega_{c},|\mu|\right), L^{2}(I)\right) \text { with }\left\|\mu^{\prime}(\cdot)\right\|_{L^{2}(I)} \equiv 1
$$

with respect to its total variation measure $|\mu|$. So $\mu$ can be represented in the following way:

$$
\mathrm{d} \mu=\mu^{\prime} \mathrm{d}|\mu|
$$

Next we introduce the space $\mathcal{C}\left(\Omega_{c}, L^{2}(I)\right)$ of vector-valued continuous functions $p: \Omega_{c} \rightarrow L^{2}(I)$. Equipped with the norm

$$
\|p\|_{\mathcal{C}\left(\Omega_{c}, L^{2}(I)\right)}=\max _{x \in \Omega_{c}}\|p(x, \cdot)\|_{L^{2}(I)}
$$

it is a separable Banach space. The dual space of $\mathcal{C}\left(\Omega_{c}, L^{2}(I)\right)$ can be characterized by $\mathcal{M}\left(\Omega_{c}, L^{2}(I)\right)$, i.e.,

$$
\mathcal{C}\left(\Omega_{c}, L^{2}(I)\right)^{*} \cong \mathcal{M}\left(\Omega_{c}, L^{2}(I)\right) .
$$

A proof is given in [17]. Next we introduce the space $L^{2}\left(I, \mathcal{M}\left(\Omega_{c}\right)\right)$. It is the space of weakly-* measurable functions $\mu: I \rightarrow \mathcal{M}\left(\Omega_{c}\right)$ which satisfy

$$
\int_{0}^{T}\|\mu(t)\|_{\mathcal{M}\left(\Omega_{c}\right)}^{2} \mathrm{~d} t<\infty
$$

where $\mathcal{M}\left(\Omega_{c}\right)$ is the space of Radon measures on $\Omega_{c}$ and $\|\cdot\|_{\mathcal{M}\left(\Omega_{c}\right)}$ is the total variation norm in $\mathcal{M}\left(\Omega_{c}\right)$. There holds

$$
\mathcal{M}\left(\Omega_{c}, L^{2}(I)\right) \hookrightarrow L^{2}\left(I, \mathcal{M}\left(\Omega_{c}\right)\right) .
$$

This follows from the facts that the embedding

$$
L^{2}\left(I, \mathcal{C}\left(\Omega_{c}\right)\right) \hookrightarrow \mathcal{C}\left(\Omega_{c}, L^{2}(I)\right)
$$

is continuous and dense and that

$$
\left(L^{2}\left(I, \mathcal{C}\left(\Omega_{c}\right)\right)\right)^{*} \cong L^{2}\left(I, \mathcal{M}\left(\Omega_{c}\right)\right)
$$

holds. Next we deal with sequential weak-* compactness in $\mathcal{M}\left(\Omega_{c}, L^{2}(I)\right)$. Let $\left\{\mu_{n}\right\}_{n \in \mathbb{N}}$ be a bounded sequence in $\mathcal{M}\left(\Omega_{c}, L^{2}(I)\right)$. Then there exists an element $\mu \in \mathcal{M}\left(\Omega_{c}, L^{2}(I)\right)$ and a subsequence $\left\{\mu_{n_{k}}\right\}_{k \in \mathbb{N}}$ with

$$
\mu_{n_{k}} \rightarrow^{*} \mu \text { in } \mathcal{M}\left(\Omega_{c}, L^{2}(I)\right) ;
$$

see, e.g., [6, Corollary 3.30]. Finally we state the following density result involving the space $\mathcal{M}\left(\Omega_{c}, H^{1}(I)\right)$ which can be constructed in the same manner as $\mathcal{M}\left(\Omega_{c}, L^{2}(I)\right)$.

Proposition 2.1. The space $\mathcal{M}\left(\Omega_{c}, H^{1}(I)\right)$ is densely embedded into $\mathcal{M}\left(\Omega_{c}, L^{2}(I)\right)$.

Copyright $@$ by SIAM. Unauthorized reproduction of this article is prohibited. 
Proof. We chose any $\mu \in \mathcal{M}\left(\Omega_{c}, L^{2}(I)\right)$ and let $\mu^{\prime} \in L^{\infty}\left(\left(\Omega_{c},|\mu|\right), L^{2}(I)\right)$ be its Radon-Nikodym derivative. Extension by zero and the Tonelli-Fubini theorem imply that $\mu^{\prime} \in L^{2}\left(\mathbb{R} \times \mathbb{R}^{d}, \mathcal{L} \otimes|\mu|\right)$ holds, where $\mathcal{L} \otimes|\mu|$ denotes the product measure consisting of the Lebesgue measure on $\mathbb{R}$ and the total variation measure $|\mu|$ extended to $\mathbb{R}^{d}$ by zero. Due to the density of $\mathcal{C}_{c}^{\infty}\left(\mathbb{R} \times \mathbb{R}^{d}\right)$ in $L^{2}\left(\mathbb{R} \times \mathbb{R}^{d}, \mathcal{L} \otimes|\mu|\right)$ (cf. [3, Corollary 4.2.2]), there exists for each $\varepsilon>0$ a function $\tilde{\mu}^{\prime} \in \mathcal{C}_{c}^{\infty}\left(\mathbb{R} \times \mathbb{R}^{d}\right)$ such that

$$
\left\|\mu^{\prime}-\tilde{\mu}^{\prime}\right\|_{L^{2}\left(\mathbb{R} \times \mathbb{R}^{d}, \mathcal{L} \otimes|\mu|\right)}<\varepsilon
$$

holds. Furthermore we define $\tilde{\mu} \in \mathcal{M}\left(\Omega_{c}, H^{1}(I)\right) \subset \mathcal{M}\left(\Omega_{c}, L^{2}(I)\right)$ by

$$
\mathrm{d} \tilde{\mu}=\left.\tilde{\mu}^{\prime}\right|_{I \times \Omega_{c}} \mathrm{~d}|\mu| .
$$

Then we use the dual formulation of the norm in $\mathcal{M}\left(\Omega_{c}, L^{2}(I)\right)$ and get

$$
\begin{aligned}
\|\mu-\tilde{\mu}\|_{\mathcal{M}\left(\Omega_{c}, L^{2}(I)\right)} & =\sup _{\|\psi\|_{\mathcal{C}\left(\Omega_{c}, L^{2}(I)\right)} \leq 1} \int_{\Omega_{c}} \int_{0}^{T} \psi(x, t)\left(\mu^{\prime}(x, t)-\tilde{\mu}^{\prime}(x, t)\right) \mathrm{d} t \mathrm{~d}|\mu| \\
& \leq c\left(\Omega_{c}\right)\left\|\mu^{\prime}-\tilde{\mu}^{\prime}\right\|_{L^{2}\left(\mathbb{R} \times \mathbb{R}^{d}, \mathcal{L} \otimes|\mu|\right)}<c\left(\Omega_{c}\right) \varepsilon,
\end{aligned}
$$

which proves the assertion.

3. Interpolation spaces. In this section we discuss the required interpolation theory of Sobolev spaces based on $L^{2}(\Omega)$. Let $A=-\Delta: D(A) \subset L^{2}(\Omega) \rightarrow L^{2}(\Omega)$ be the Laplace operator with the dense domain

$$
D(A)=\left\{v \in L^{2}(\Omega)|v|_{\partial \Omega}=0, A v \in L^{2}(\Omega)\right\} .
$$

It is an unbounded, positive, self-adjoint, and therefore closed operator with a bounded inverse $A^{-1}: L^{2}(\Omega) \rightarrow D(A)$. The fractional powers $A^{\theta}$ of $A$ with domains $D\left(A^{\theta}\right)$ can be defined for $\theta \geq 0$ as in [2, Part 2, section 1.5]. In the following sections we will use the notation

$$
V^{3}=D\left(A^{3 / 2}\right), \quad V^{2}=D(A), \quad V=D\left(A^{1 / 2}\right), \quad H=L^{2}(\Omega) .
$$

The space $D\left(A^{\theta}\right)$ is a Banach space when endowed with the graph norm

$$
v \mapsto\|v\|_{H}+\left\|A^{\theta} v\right\|_{H}, \quad v \in D\left(A^{\theta}\right) .
$$

The functional

$$
v \mapsto\left\|A^{\theta} v\right\|_{H}, \quad v \in D\left(A^{\theta}\right),
$$

defines an equivalent norm on $D\left(A^{\theta}\right)$ and we set $\|\cdot\|_{D\left(A^{\theta}\right)}:=\left\|A^{\theta} \cdot\right\|_{H}$. For $0 \leq \theta \leq 1$ the spaces $D\left(A^{\theta}\right)$ can be represented using complex interpolation spaces $[\cdot, \cdot]_{1-\theta}$ in the following form:

$$
D\left(A^{\theta}\right)=\left[V^{2}, H\right]_{1-\theta} ;
$$

see [2, Proposition 6.1, Part 2, section 1]. The spaces $D\left(A^{\theta}\right)$ can also be characterized as the Sobolev spaces $H^{2 \theta}(\Omega)$ with additional boundary conditions as follows:

$$
D\left(A^{\theta}\right)=\left\{\begin{aligned}
& v \in H^{2 \theta}(\Omega):\left.v\right|_{\partial \Omega}=0 \text { if } 1 \geq \theta>1 / 4 \\
& v \cdot \zeta^{-1 / 2} \in H \text { if } \theta=1 / 4
\end{aligned}\right\},
$$

Copyright $\odot$ by SIAM. Unauthorized reproduction of this article is prohibited. 
where $\zeta \in \mathcal{C}^{\infty}(\bar{\Omega})$ vanishes on $\partial \Omega$ of the order of $\operatorname{dist}(x, \partial \Omega)$ (distance from $x$ to $\partial \Omega$ ). In particular there holds that

$$
D\left(A^{\theta}\right)= \begin{cases}H^{2 \theta}(\Omega) \cap V, & 3 / 4<\theta \leq 1, \\ H_{0}^{2 \theta}(\Omega), & 1 / 4<\theta \leq 3 / 4, \\ H_{00}^{1 / 2}(\Omega), & \theta=1 / 4, \\ H^{2 \theta}(\Omega), & 0 \leq \theta<1 / 4\end{cases}
$$

e.g., see $[26$, Chapter 1 , Theorems $11.5,11.6,11.7]$. The space $H_{00}^{1 / 2}(\Omega)$ is given by

$$
H_{00}^{1 / 2}(\Omega)=\left\{v \in H^{1 / 2}(\Omega) \mid \zeta^{-1 / 2} v \in H\right\}
$$

and therefore we have $H_{00}^{1 / 2}(\Omega) \subset H^{1 / 2}(\Omega)$. The space $D\left(A^{\theta}\right)^{*}$ is a Banach space when endowed with the operator norm

$$
\|w\|_{D\left(A^{\theta}\right)^{*}}=\sup _{\|v\|_{D\left(A^{\theta}\right)} \leq 1}\langle w, v\rangle_{D\left(A^{\theta}\right)^{*}, D\left(A^{\theta}\right)}, \quad w \in D\left(A^{\theta}\right)^{*} .
$$

The topological dual operator of $A^{\theta}$ has the following property $\left(A^{\theta}\right)^{*}: H \rightarrow$ $D\left(A^{\theta}\right)^{*}$ and

$$
\left(y, A^{\theta} v\right)_{H}=\left\langle\left(A^{\theta}\right)^{*} y, v\right\rangle_{D\left(A^{\theta}\right)^{*}, D\left(A^{\theta}\right)}, \quad y \in H, v \in D\left(A^{\theta}\right) .
$$

Since $A^{\theta}$ is an isomorphism from $D\left(A^{\theta}\right)$ to $H$, the operator $\left(A^{\theta}\right)^{*}$ is also an isomorphism with the inverse $\left(A^{\theta}\right)^{-*}: D\left(A^{\theta}\right)^{*} \rightarrow H$. This also means that the equation

$$
\left(A^{\theta}\right)^{*} y=w \text { in } D\left(A^{\theta}\right)^{*}
$$

has a unique solution $y=\left(A^{\theta}\right)^{-*} w \in H$ for every $w \in D\left(A^{\theta}\right)^{*}$. Using this dual equation we can estimate

$$
\begin{aligned}
\|w\|_{D\left(A^{\theta}\right)^{*}} & =\sup _{\|v\|_{D\left(A^{\theta}\right)} \leq 1}\langle w, v\rangle_{D\left(A^{\theta}\right)^{*}, D\left(A^{\theta}\right)}=\sup _{\|v\|_{D\left(A^{\theta}\right)} \leq 1}\left\langle\left(A^{\theta}\right)^{*} y, v\right\rangle_{D\left(A^{\theta}\right)^{*}, D\left(A^{\theta}\right)} \\
& \leq\|y\|_{H}=\left\|\left(A^{\theta}\right)^{-*} w\right\|_{H}
\end{aligned}
$$

and

$$
\left\|\left(A^{\theta}\right)^{-*} w\right\|_{H}=\frac{\left(y, A^{\theta} A^{-\theta} y\right)_{H}}{\|y\|_{H}}=\frac{\left\langle\left(A^{\theta}\right)^{*} y, A^{-\theta} y\right\rangle_{D\left(A^{\theta}\right)^{*}, D\left(A^{\theta}\right)}}{\left\|A^{-\theta} y\right\|_{D\left(A^{\theta}\right)}} \leq\|w\|_{D\left(A^{\theta}\right)^{*}},
$$

which implies

$$
\|w\|_{D\left(A^{\theta}\right)^{*}}=\left\|\left(A^{\theta}\right)^{-*} w\right\|_{H}
$$

The duality pairing $\langle\cdot, \cdot\rangle_{D\left(A^{\theta}\right), D\left(A^{\theta}\right)^{*}}$ can be expressed using $\left(A^{\theta}\right)^{-*}$ in the following form:

$$
\begin{aligned}
\langle w, v\rangle_{D\left(A^{\theta}\right)^{*}, D\left(A^{\theta}\right)} & =\left\langle\left(A^{\theta}\right)^{*} y, v\right\rangle_{D\left(A^{\theta}\right)^{*}, D\left(A^{\theta}\right)}=\left(y, A^{\theta} v\right)_{H} \\
& =\left(\left(A^{\theta}\right)^{-*} w, A^{\theta} v\right)_{H} \text { for } v \in D\left(A^{\theta}\right), w \in D\left(A^{\theta}\right)^{*} .
\end{aligned}
$$

In the following we give a characterization of $D\left(A^{\theta}\right)^{*}$ by Sobolev spaces with negative indices. According to [26, Chapter 1, Theorem 6.2]

$$
D\left(A^{\theta}\right)^{*}=\left[H, V^{2 *}\right]_{\theta}, \quad 0 \leq \theta \leq 1,
$$

Copyright $@$ by SIAM. Unauthorized reproduction of this article is prohibited. 
holds, and more concretely

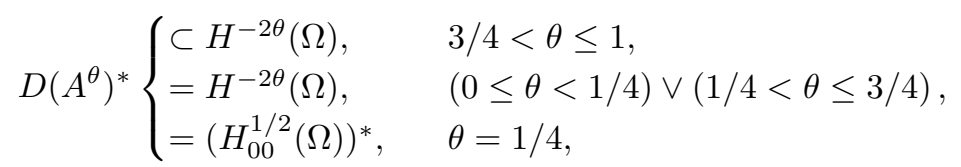

e.g., [26, Chapter 1, Theorem 12.2], where $H^{-1 / 2}(\Omega) \subset H_{00}^{1 / 2}(\Omega)^{*}$. Using (3.2) it follows by classical theory for fractional Sobolev spaces that

$$
D\left(A^{\theta}\right) \hookrightarrow \mathcal{C}\left(\Omega_{c}\right)
$$

holds for $\theta>d / 4$ and therefore also

$$
\mathcal{M}\left(\Omega_{c}\right) \hookrightarrow D\left(A^{\theta}\right)^{*}
$$

for $\theta>d / 4$. Finally we define the space

$$
X_{\theta}:= \begin{cases}D\left(A^{\theta}\right), & 0 \leq \theta \leq 3 / 2, \\ D\left(A^{|\theta|}\right)^{*}, & -3 / 2 \leq \theta<0,\end{cases}
$$

which will be convenient in our analysis. Its norm is denoted by $\|\cdot\|_{X_{\theta}}$ and is given by the norm of the underlying space. The duality pairing is denoted by $\langle\cdot, \cdot\rangle_{X_{\theta}, X_{-\theta}}$.

4. Well-posedness of the state equation and adjoint equation. In this section we introduce the weak and very weak formulations of the linear wave equation

$$
\left\{\begin{aligned}
\partial_{t t} y-\Delta y & =f & & \text { in } I \times \Omega \\
y & =0 & & \text { on } I \times \partial \Omega \\
\left(y, \partial_{t} y\right) & =\left(y_{0}, y_{1}\right) & & \text { in }\{0\} \times \Omega .
\end{aligned}\right.
$$

(With no loss of generality we set the constant wave speed to $c=1$.) We show existence, uniqueness, and regularity of solutions of (4.1) and its dual counterpart for different regularity classes of data. First we introduce the notion of a weak solution of (4.1).

Definition 4.1. Let $\left(f, y_{0}, y_{1}\right) \in L^{1}(I, H) \times V \times H$. A function

$$
y \in L^{2}(I, V) \cap W^{2,1}\left(I, V^{*}\right)
$$

is called a weak solution of (4.1) if it satisfies

$$
\begin{aligned}
\int_{0}^{T}\left\langle\partial_{t t} y, v\right\rangle_{V^{*}, V}+(\nabla y, \nabla v)_{H} \mathrm{~d} t & =\int_{0}^{T}(f, v)_{H} \mathrm{~d} t \quad \forall v \in H^{1}(I, V), \\
y(0) & =y_{0}, \\
\partial_{t} y(0) & =y_{1} .
\end{aligned}
$$

Remark 4.2. The values of $y(0)$ and $\partial_{t} y(0)$ are well defined since

$$
L^{2}(I, V) \cap W^{2,1}\left(I, V^{*}\right) \hookrightarrow \mathcal{C}(\bar{I}, H) \cap \mathcal{C}^{1}\left(\bar{I}, V^{*}\right) .
$$

Next we deal with the existence, uniqueness, and regularity of a weak solution for the linear wave equation (4.1). For that we need the following Gronwall lemma.

Copyright $@$ by SIAM. Unauthorized reproduction of this article is prohibited. 
Lemma 4.3. Suppose

$$
v(t)^{2} \leq c^{2}+2 \int_{0}^{t} \psi(s) v(s) \mathrm{d} s \quad \text { a.e. } t \in I,
$$

where $c \in \mathbb{R}, \psi \in L^{1}(I)$ with $\psi(t) \geq 0$, and $v \in L^{\infty}(I)$ with $v(t) \geq 0$. Then there holds

$$
v(t) \leq c+\int_{0}^{t} \psi(s) \mathrm{d} \text { s a.e. } t \in I .
$$

Proof. The proof can be found, e.g., in [13, Theorem 5]. time.

Furthermore we require the following lemma concerning integration by parts in

LEMma 4.4. Let $Z$ be a separable Hilbert space and $y \in W^{1,1}(I, Z)$ as well as $\phi \in W^{1,1}\left(I, Z^{*}\right)$. Then there holds

$$
\int_{0}^{T}\left\langle y, \partial_{t} \phi\right\rangle_{Z, Z^{*}} \mathrm{~d} t=-\int_{0}^{T}\left\langle\partial_{t} y, \phi\right\rangle_{Z, Z^{*}} \mathrm{~d} t+\left.\left\langle y, \partial_{t} \phi\right\rangle_{Z, Z^{*}}\right|_{0} ^{T} .
$$

Proof. According to [5, Corollary A.4], the mapping $B: t \mapsto\langle y(t), \phi(t)\rangle_{Z, Z^{*}}$ belongs to $W^{1,1}(I)$. Owing to the fundamental theorem of calculus for $W^{1,1}(I)$ functions, we get

$$
\int_{0}^{T} \partial_{t} B \mathrm{~d} t=\left.\langle y, \phi\rangle_{Z, Z^{*}}\right|_{0} ^{T}
$$

Moreover, we have

$$
\partial_{t} B=\left\langle\partial_{t} y, \phi\right\rangle_{Z, Z^{*}}+\left\langle y, \partial_{t} \phi\right\rangle_{Z, Z^{*}}
$$

Combining these two facts implies the result.

THEOREM 4.5. The following existence, uniqueness, and regularity results hold true:

1. (Standard regularity) Let $\left(f, y_{0}, y_{1}\right) \in L^{r}(I, H) \times V \times H$ for $r \in[1, \infty]$. Then there exists a unique weak solution y of (4.1) satisfying the following regularity property:

$$
y \in \mathcal{C}(\bar{I}, V) \cap \mathcal{C}^{1}(\bar{I}, H) \cap W^{2, r}\left(I, V^{*}\right) .
$$

Furthermore there exists a constant $c>0$ such that

$$
\begin{array}{r}
\|y\|_{\mathcal{C}(\bar{I}, V)}+\left\|\partial_{t} y\right\|_{\mathcal{C}(\bar{I}, H)}+\left\|\partial_{t t} y\right\|_{L^{r}\left(I, V^{*}\right)} \\
\leq c\left(\|f\|_{L^{r}(I, H)}+\left\|y_{0}\right\|_{V}+\left\|y_{1}\right\|_{H}\right)
\end{array}
$$

holds.

2. (Higher regularity) Let $\left(f, y_{0}, y_{1}\right) \in L^{r}(I, V) \times V^{2} \times V$ for $r \in[1, \infty]$. Then the solution of (4.1) satisfies

$$
y \in \mathcal{C}\left(\bar{I}, V^{2}\right) \cap \mathcal{C}^{1}(\bar{I}, V) \cap W^{2, r}(I, H)
$$

and there exists a constant $c>0$ such that

$$
\begin{array}{r}
\|y\|_{\mathcal{C}\left(\bar{I}, V^{2}\right)}+\left\|\partial_{t} y\right\|_{\mathcal{C}(\bar{I}, V)}+\left\|\partial_{t t} y\right\|_{L^{r}(I, H)} \\
\leq c\left(\|f\|_{L^{r}(I, V)}+\left\|y_{0}\right\|_{V^{2}}+\left\|y_{1}\right\|_{V}\right)
\end{array}
$$

is fulfilled.

Copyright $@$ by SIAM. Unauthorized reproduction of this article is prohibited. 
Proof. Uniqueness is shown first. Let $y \in L^{2}(I, V) \cap W^{2,1}\left(I, V^{*}\right)$ be a weak solution of (4.1) for $y_{0}=y_{1}=f=0$. Then we define the function

$$
\psi(t, x)= \begin{cases}-\int_{t}^{s} y(\sigma, x) \mathrm{d} \sigma, & t \leq s \\ 0 & \text { else }\end{cases}
$$

for some $0<s \leq T$. In particular, $\psi \in H^{1}(I, V)$. So we can test (4.1) with $\psi$ and get

$$
\int_{0}^{s}\left\langle\partial_{t t} y,-\int_{t}^{s} y(\sigma) \mathrm{d} \sigma\right\rangle_{V^{*}, V}-\left(\nabla y, \nabla \int_{t}^{s} y(\sigma) \mathrm{d} \sigma\right)_{H} \mathrm{~d} t=0 .
$$

Partial integration in time, which is permissible according to Lemma 4.4, and $\partial_{t} \psi=y$ on $(0, s)$ yield

$$
\int_{0}^{s}-\left\langle\partial_{t} y, y\right\rangle_{V^{*}, V}+\left(\nabla \partial_{t} \psi, \nabla \psi\right)_{H} \mathrm{~d} t=0
$$

Then $y \in L^{2}(I, V) \cap H^{1}\left(I, V^{*}\right) \hookrightarrow \mathcal{C}(\bar{I}, H)$ and $\psi \in H^{1}(I, V) \hookrightarrow \mathcal{C}(\bar{I}, V)$ yield

$$
\|y(s)\|_{H}^{2}+\|\psi(0)\|_{V}^{2}=0 \quad \forall s \in \bar{I} .
$$

This implies $y \equiv 0$ and therefore the uniqueness of the solution $y$. In order to show existence we approximate the data by smooth functions. The corresponding smooth solutions $y$ satisfy the estimates

$$
\|y\|_{\mathcal{C}(\bar{I}, V)}+\left\|\partial_{t} y\right\|_{\mathcal{C}(\bar{I}, H)}+\left\|\partial_{t t} y\right\|_{L^{r}\left(I, V^{*}\right)} \leq c_{1}\left(\left\|y_{0}\right\|_{V}+\left\|y_{1}\right\|_{H}+\|f\|_{L^{r}(I, H)}\right)
$$

and

$$
\|y\|_{\mathcal{C}\left(\bar{I}, V^{2}\right)}+\left\|\partial_{t} y\right\|_{\mathcal{C}(\bar{I}, V)}+\left\|\partial_{t t} y\right\|_{L^{r}(I, H)} \leq c_{2}\left(\left\|y_{0}\right\|_{V^{2}}+\left\|y_{1}\right\|_{V}+\|f\|_{L^{r}(I, V)}\right)
$$

for some $c_{1}, c_{2}>0$ independent of $y$ and the data. Indeed, the estimates for $y$ and $\partial_{t} y$ can be derived by testing the equation $\partial_{t t} y-\Delta y=f$ with $\partial_{t} y$, respectively, $-\Delta \partial_{t} y$, and by using Lemma 4.3. The estimate for $\partial_{t t} y$ follows from

$$
\left\|\partial_{t t} y\right\|_{L^{r}\left(I, V^{*}\right)}=\|\Delta y+f\|_{L^{r}\left(I, V^{*}\right)} \leq c_{3}\left(\left\|y_{0}\right\|_{V}+\left\|y_{1}\right\|_{H}+\|f\|_{L^{r}(I, H)}\right)
$$

for some $c_{3}>0$ independent of $y$ and the data. The estimate for $\left\|\partial_{t t} y\right\|_{L^{r}(I, H)}$ can be derived in a similar fashion. Then a standard density argument yields the existence of a weak solution with the claimed regularity properties.

Remark 4.6. Due to the time reversibility of the linear wave equation, Theorem 4.5 can be applied to the adjoint wave equation

$$
\left\{\begin{aligned}
\partial_{t t} p-\Delta p & =\phi & & \text { in } I \times \Omega, \\
p & =0 & & \text { on } I \times \partial \Omega, \\
\left(p, \partial_{t} p\right) & =\left(p_{0}, p_{1}\right) & & \text { in }\{T\} \times \Omega
\end{aligned}\right.
$$

and yields existence, uniqueness, and the same regularity results for $p$ as for the solution of (4.1).

In the remainder of the section we turn our attention to the case with a more irregular source term $f$.

Copyright $@$ by SIAM. Unauthorized reproduction of this article is prohibited. 
Definition 4.7. For $\left(f, y_{0}, y_{1}\right) \in L^{1}\left(I, V^{2 *}\right) \times V^{*} \times V^{2 *}$ a function $y$ with

$$
\left(y, y(T), \partial_{t} y(T)\right) \in L^{\infty}\left(I, V^{*}\right) \cap W^{2,1}\left(I, V^{3 *}\right) \times V^{*} \times V^{2 *}
$$

is called a very weak solution of (4.1), if it satisfies the equation

$$
\begin{aligned}
\int_{0}^{T}\langle y, \phi\rangle_{V^{*}, V} \mathrm{~d} t-\left\langle y(T), p_{1}\right\rangle_{V^{*}, V}+\left\langle\partial_{t} y(T), p_{0}\right\rangle_{V^{2 *}, V^{2}} \\
\quad=\int_{0}^{T}\langle f, p\rangle_{V^{2 *}, V^{2}} \mathrm{~d} t-\left\langle y_{0}, \partial_{t} p(0)\right\rangle_{V^{*}, V}+\left\langle y_{1}, p(0)\right\rangle_{V^{2 *}, V^{2}}
\end{aligned}
$$

for all $\left(\phi, p_{1}, p_{0}\right) \in L^{1}(I, V) \times V \times V^{2}$, where $p\left(\phi, p_{1}, p_{0}\right) \in \mathcal{C}\left(\bar{I}, V^{2}\right) \cap \mathcal{C}^{1}(\bar{I}, V)$ is the solution of (4.4).

We remark that very weak solutions of linear PDEs are also called solutions by transposition; see, e.g., [26]. Next, we show the existence and uniqueness of a very weak solution.

Proposition 4.8. Let $\left(f, y_{0}, y_{1}\right) \in L^{r}\left(I, V^{2 *}\right) \times V^{*} \times V^{2 *}$ for $r \in[1, \infty]$. Then there exists a unique very weak solution $y$ of (4.1) which has the following regularity property:

$$
y \in \mathcal{C}\left(\bar{I}, V^{*}\right) \cap \mathcal{C}^{1}\left(I, V^{2 *}\right) \cap W^{2, r}\left(I, V^{3 *}\right) .
$$

Furthermore there exists a constant $c>0$ such that

$$
\begin{array}{r}
\|y\|_{\mathcal{C}\left(\bar{I}, V^{*}\right)}+\left\|\partial_{t} y\right\|_{\mathcal{C}\left(\bar{I}, V^{2 *}\right)}+\left\|\partial_{t t} y\right\|_{L^{r}\left(I, V^{3 *}\right)} \\
\leq c\left(\|f\|_{L^{r}\left(I, V^{2 *}\right)}+\left\|y_{0}\right\|_{V^{*}}+\left\|y_{1}\right\|_{V^{2 *}}\right)
\end{array}
$$

holds.

Proof. Uniqueness is shown first. Suppose that $y$ is the very weak solution of (4.1) for the datum $f=y_{0}=y_{1}=0$. Then we have

$$
\int_{0}^{T}\langle y, \phi\rangle_{V^{*}, V} \mathrm{~d} t-\left\langle y(T), p_{1}\right\rangle_{V^{*}, V}+\left\langle\partial_{t} y(T), p_{0}\right\rangle_{V^{2 *}, V^{2}}=0
$$

for all $\left(\phi, p_{1}, p_{0}\right) \in V \times V \times V^{2}$. This implies $y=y(T)=\partial_{t} y(T)=0$. The proof of existence is based on approximation of the data; cf. [26, Chapter 3, Theorem 9.3]. For that purpose we introduce the following approximating sequences:

- $\left\{f_{n}\right\}_{n \in \mathbb{N}} \subset \mathcal{C}_{c}^{\infty}(I \times \Omega)$ with $f_{n} \rightarrow f$ in $L^{1}\left(I, V^{2 *}\right)$,

- $\left\{y_{0, n}\right\}_{n \in \mathbb{N}} \subset \mathcal{C}_{c}^{\infty}(\Omega)$ with $y_{0, n} \rightarrow y_{0}$ in $V^{*}$,

- $\left\{y_{1, n}\right\}_{n \in \mathbb{N}} \subset \mathcal{C}_{c}^{\infty}(\Omega)$ with $y_{1, n} \rightarrow y_{1}$ in $V^{2 *}$.

Then we consider the auxiliary problem

$$
\left\{\begin{aligned}
\partial_{t t} y_{n}-\Delta y_{n} & =f_{n} & & \text { in } I \times \Omega, \\
y_{n} & =0 & & \text { on } I \times \partial \Omega, \\
\left(y_{n}, \partial_{t} y_{n}\right) & =\left(y_{0, n}, y_{1, n}\right) & & \text { in }\{0\} \times \Omega,
\end{aligned}\right.
$$

which has a unique smooth solution $y_{n}$. Subsequently we multiply $\partial_{t t} y_{n}-\Delta y_{n}=f_{n}$ with $A^{-2} \partial_{t} y_{n}$, integrate over $(0, t) \times \Omega$ for some $t \in(0, T]$, and see

$$
\begin{gathered}
\int_{0}^{t}\left(\partial_{t t} y_{n}, A^{-2} \partial_{t} y_{n}\right)_{H} \mathrm{~d} t=\int_{0}^{t}\left(A^{-1} \partial_{t t} y_{n}, A^{-1} \partial_{t} y_{n}\right)_{H} \mathrm{~d} t \\
=\frac{1}{2}\left(\left\|\partial_{t} y_{n}(t)\right\|_{V^{2 *}}^{2}-\left\|y_{1, n}\right\|_{V^{2 *}}^{2}\right),
\end{gathered}
$$

Copyright $@$ by SIAM. Unauthorized reproduction of this article is prohibited. 


$$
\begin{aligned}
\int_{0}^{t}( & \left.-\Delta y_{n}, A^{-2} \partial_{t} y_{n}\right)_{H} \mathrm{~d} t=\int_{0}^{t}\left(y_{n}, A^{-1} \partial_{t} y_{n}\right)_{H} \mathrm{~d} t \\
& =\int_{0}^{t}\left(\nabla A^{-1} y_{n}, \nabla A^{-1} \partial_{t} y_{n}\right)_{H} \mathrm{~d} t=\frac{1}{2}\left(\left\|y_{n}(t)\right\|_{V^{*}}^{2}-\left\|y_{0, n}\right\|_{V^{*}}^{2}\right)
\end{aligned}
$$

as well as

$$
\int_{0}^{t}\left(f_{n}, A^{-2} \partial_{t} y_{n}\right)_{H} \mathrm{~d} t=\int_{0}^{t}\left(A^{-1} f_{n}, A^{-1} \partial_{t} y_{n}\right)_{H} \mathrm{~d} t \leq \int_{0}^{t}\left\|f_{n}\right\|_{V^{2 *}}\left\|\partial_{t} y_{n}\right\|_{V^{2 *}} \mathrm{~d} t .
$$

Applying the Gronwall-type Lemma 4.3 yields the following estimate:

$$
\left\|y_{n}\right\|_{\mathcal{C}\left(\bar{I}, V^{*}\right)}+\left\|\partial_{t} y_{n}\right\|_{\mathcal{C}\left(\bar{I}, V^{2 *}\right)} \leq c\left(\left\|f_{n}\right\|_{L^{1}\left(I, V^{2 *}\right)}+\left\|y_{0, n}\right\|_{V^{*}}+\left\|y_{1, n}\right\|_{V^{2 *}}\right) .
$$

Next we derive an estimate for $\partial_{t t} y_{n}$. We get

$$
\begin{aligned}
& \int_{0}^{T}\left(\partial_{t t} y_{n}, \psi\right)_{H} \mathrm{~d} t=\int_{0}^{T}\left(f_{n}+\Delta y_{n}, \psi\right)_{H} \mathrm{~d} t \\
&=\int_{0}^{T}\left(f_{n}, \psi\right)_{H}+\left(y_{n}, \Delta \psi\right)_{H} \mathrm{~d} t \leq c\left(\left\|f_{n}\right\|_{L^{r}\left(I, V^{2 *}\right)}+\left\|y_{n}\right\|_{\mathcal{C}\left(\bar{I}, V^{*}\right)}\right)\|\psi\|_{L^{s}\left(I, V^{3}\right)}
\end{aligned}
$$

for any $\psi \in L^{s}\left(I, V^{3}\right)$ with $r^{-1}+s^{-1}=1$, which implies

$$
\begin{aligned}
\left\|\partial_{t t} y_{n}\right\|_{L^{r}\left(I, V^{3 *}\right)} & \leq c\left(\left\|y_{n}\right\|_{\mathcal{C}\left(\bar{I}, V^{*}\right)}+\left\|f_{n}\right\|_{L^{r}\left(I, V^{2 *}\right)}\right) \\
& \leq c\left(\left\|f_{n}\right\|_{L^{r}\left(I, V^{2 *}\right)}+\left\|y_{0, n}\right\|_{V^{*}}+\left\|y_{1, n}\right\|_{V^{2 *}}\right) .
\end{aligned}
$$

This estimate implies that $\left\{y_{n}\right\}_{n \in \mathbb{N}}$ is a Cauchy sequence in

$$
\mathcal{C}\left(\bar{I}, V^{*}\right) \cap \mathcal{C}^{1}\left(\bar{I}, V^{2 *}\right) \cap W^{2, r}\left(I, V^{3 *}\right) .
$$

So there exist a $y$ with $y_{n} \rightarrow y$ in $\mathcal{C}\left(\bar{I}, V^{*}\right) \cap \mathcal{C}^{1}\left(\bar{I}, V^{2 *}\right) \cap W^{2, r}\left(I, V^{3 *}\right)$. Next we multiply $\partial_{t t} y_{n}-\Delta y_{n}=f_{n}$ with the solution $p \in \mathcal{C}\left(\bar{I}, V^{2}\right) \cap \mathcal{C}^{1}(\bar{I}, V)$ of (4.4) for any $\left(\phi, p_{1}, p_{0}\right) \in L^{1}(I, V) \times V \times V^{2}$ and integrate over $I \times \Omega$. Integration by parts in time and in space yields

$$
\begin{aligned}
\int_{0}^{T}\left(y_{n}, \phi\right)_{H} \mathrm{~d} t-\left(y_{n}(T), p_{1}\right)_{H}+\left(\partial_{t} y_{n}(T), p_{0}\right)_{H} \\
\quad=\int_{0}^{T}\left(f_{n}, p\right)_{H} \mathrm{~d} t-\left(y_{0, n}, \partial_{t} p(0)\right)_{H}+\left(y_{1, n}, p(0)\right)_{H} .
\end{aligned}
$$

After passing to the limit in (4.9) we see that $y$ fulfills (4.5). Moreover $y$ satisfies

$$
\begin{array}{r}
\|y\|_{\mathcal{C}\left(\bar{I}, V^{*}\right)}+\left\|\partial_{t} y\right\|_{\mathcal{C}\left(\bar{I}, V^{2 *}\right)}+\left\|\partial_{t t} y\right\|_{L^{r}\left(I, V^{3 *}\right)} \\
\leq c\left(\|f\|_{L^{r}\left(I, V^{2 *}\right)}+\left\|y_{0}\right\|_{V^{*}}+\left\|y_{1}\right\|_{V^{2 *}}\right) .
\end{array}
$$

Now we can achieve the following regularity results by interpolation of the solution operator $S:\left(f, y_{0}, y_{1}\right) \mapsto\left(y, \partial_{t} y, \partial_{t t} y\right)$ between the results of Theorem 4.5 and Proposition 4.8 .

Copyright $@$ ( ) by SIAM. Unauthorized reproduction of this article is prohibited. 
Corollary 4.9. Let $\theta \in[-1,1 / 2]$. If $\left(f, y_{0}, y_{1}\right) \in L^{r}\left(I, X_{\theta}\right) \times X_{\theta+1 / 2} \times X_{\theta}$ for $r \in[1, \infty]$. Then there exists a unique solution $y$ of (4.1) which satisfies

$$
y \in \mathcal{C}\left(\bar{I}, X_{\theta+1 / 2}\right) \cap \mathcal{C}^{1}\left(\bar{I}, X_{\theta}\right) \cap W^{2, r}\left(I, X_{\theta-1 / 2}\right) .
$$

Furthermore there exists a constant $c>0$ such that

$$
\begin{array}{r}
\|y\|_{\mathcal{C}\left(\bar{I}, X_{\theta+1 / 2}\right)}+\left\|\partial_{t} y\right\|_{\mathcal{C}\left(\bar{I}, X_{\theta}\right)}+\left\|\partial_{t t} y\right\|_{L^{r}\left(I, X_{\theta-1 / 2}\right)} \\
\leq c\left(\|f\|_{L^{r}\left(I, X_{\theta}\right)}+\left\|y_{0}\right\|_{X_{\theta+1 / 2}}+\left\|y_{1}\right\|_{X_{\theta}}\right)
\end{array}
$$

holds.

Remark 4.10. The results of Corollary 4.9 hold also for the dual equation (4.4).

Finally we consider the state equation (1.1) with controls from $\mathcal{M}\left(\Omega_{c}, L^{2}(I)\right)$. We recall (2.2) and (3.4) which imply the embedding

$$
\mathcal{M}\left(\Omega_{c}, L^{2}(I)\right) \hookrightarrow L^{2}\left(I, \mathcal{M}\left(\Omega_{c}\right)\right) \hookrightarrow L^{2}\left(I, X_{-d / 4-\varepsilon}\right)
$$

for any $3 / 4>\varepsilon>0$. Then we can use Corollary 4.9 to establish the well-posedness of (1.1) in the following sense.

Corollary 4.11. For any $\left(u, y_{0}, y_{1}\right) \in \mathcal{M}\left(\Omega_{c}, L^{2}(I)\right) \times X_{(2-d) / 4-\varepsilon} \times X_{-d / 4-\varepsilon}$, $0<\varepsilon<3 / 4$, there exists a unique very weak solution $y$ of (1.1) which additionally satisfies

$$
y \in \mathcal{C}\left(\bar{I}, X_{(2-d) / 4-\varepsilon}\right) \cap \mathcal{C}^{1}\left(\bar{I}, X_{-d / 4-\varepsilon}\right) \cap H^{2}\left(I, X_{-(2+d) / 4-\varepsilon}\right)
$$

and there exists a constant $c>0$

$$
\begin{aligned}
\|y\|_{\mathcal{C}\left(\bar{I}, X_{(2-d) / 4-\varepsilon}\right)}+\left\|\partial_{t} y\right\|_{\mathcal{C}\left(\bar{I}, X_{-d / 4-\varepsilon}\right)}+\left\|\partial_{t t} y\right\|_{L^{2}\left(I, X_{-(2+d) / 4-\varepsilon}\right)} & \\
& \leq c\left(\|u\|_{\mathcal{M}\left(\Omega_{c}, L^{2}(I)\right)}+\left\|y_{0}\right\|_{X_{(2-d) / 4-\varepsilon}}+\left\|y_{1}\right\|_{X_{-d / 4-\varepsilon}}\right)
\end{aligned}
$$

According to the last corollary there holds $y \in \mathcal{C}\left(\bar{I}, L^{2}(\Omega)\right) \cap \mathcal{C}^{1}\left(\bar{I}, H^{-1}(\Omega)\right)$ only for $d=1$.

Since the embedding (4.12) is used for the proof of Corollary 4.11 it is not clear if the regularity results of Corollary 4.11 are sharp. In the next section we will see that regularity is lost by using the embedding (4.12).

5. Improved regularity results for the state and adjoint equation. In this section we will establish higher regularity of the state variable $y$ using explicitly properties of the control space $\mathcal{M}\left(\Omega_{c}, L^{2}(I)\right)$ and not through the embedding (4.12). To do so we will first establish $\mathcal{C}\left(\Omega_{c}, L^{2}(I)\right)$ regularity of the solution $p$ of the dual wave equation (4.4) for certain regularity classes of data. These results can be used to show the mentioned higher regularity of the primal variable. In the following we will invoke and recap some regularity results for the primal equation (4.1) with a specific source term $f(t)=h(t) \delta_{x_{0}}$ with $x_{0} \in \Omega_{c}$ and $h \in L^{2}(I)$ which were proven in [28, 24] for dimensions $d=1,2,3$ and in [25] for $d=3$. These results will play an important role in the proof of the $\mathcal{C}\left(\Omega_{c}, L^{2}(I)\right)$ regularity of $p$. In other words, we consider the following equation:

$$
\left\{\begin{array}{rlrl}
\partial_{t t} y-\Delta y & =h \delta_{x_{0}} & & \text { in } I \times \Omega, \\
y=0 & & \text { on } I \times \partial \Omega \\
\left(y, \partial_{t} y\right) & =0 & & \text { in }\{0\} \times \Omega .
\end{array}\right.
$$

Copyright (c) by SIAM. Unauthorized reproduction of this article is prohibited. 
Corollary 4.11 implies that a unique very weak solution $y$ of (5.1) exists and has at least the regularity

$$
y \in \mathcal{C}\left(\bar{I}, X_{-(d-2) / 4-\varepsilon}\right) \cap \mathcal{C}^{1}\left(\bar{I}, X_{-d / 4-\varepsilon}\right) \cap H^{2}\left(I, X_{-(d+2) / 4-\varepsilon}\right) .
$$

But this result is not optimal. In the remainder of this paper we fix the following notation:

$$
\theta_{d}=\frac{1-d}{4}
$$

Proposition 5.1. Let $h \in L^{2}(I), x_{0} \in \Omega_{c}$ and let $y$ be the very weak solution of (5.1). Then

$$
y \in \mathcal{C}\left(\bar{I}, X_{\theta_{d}+1 / 2}\right) \cap \mathcal{C}^{1}\left(\bar{I}, X_{\theta_{d}}\right) \cap H^{2}\left(I, X_{\theta_{d}-1 / 2}\right)
$$

holds. Moreover there exists a constant $c\left(\Omega_{c}\right)>0$ independent of $y, h$, and $x_{0}$ such that

$$
\|y\|_{\mathcal{C}\left(\bar{I}, X_{\theta_{d}+1 / 2}\right)}+\left\|\partial_{t} y\right\|_{\mathcal{C}\left(\bar{I}, X_{\theta_{d}}\right)}+\left\|\partial_{t t} y\right\|_{L^{2}\left(I, X_{\theta_{d}}-1 / 2\right)} \leq c\|h\|_{L^{2}(I)}
$$

holds.

Proof. This result is essentially proved in [28, Theorem 2.1], without detailing the dependence on $x_{0}$. But since the dependence of the constant $c$ in (5.2) on $x_{0}$ plays an important role for the following consideration, we repeat the important steps of the proof in order to investigate the role of $x_{0}$. We first consider the free space problem

$$
\left\{\begin{aligned}
\partial_{t t} \psi-\Delta \psi & =h \delta_{x_{0}} & & \text { in } \mathbb{R}^{n} \times I, \\
\left(\psi, \partial_{t} \psi\right) & =0 & & \text { on }\{0\} \times \mathbb{R}^{n} .
\end{aligned}\right.
$$

In [28] it is shown using the Laplace transform in time and the Fourier transform in space that (5.3) admits a solution $\psi$ with the regularity property

$$
\psi \in \mathcal{C}\left(\bar{I},\left[H^{2}\left(\mathbb{R}^{n}\right), L^{2}\left(\mathbb{R}^{n}\right)\right]_{1 / 2-\theta_{d}}\right) \cap \mathcal{C}^{1}\left(\bar{I},\left[L^{2}\left(\mathbb{R}^{n}\right), H^{-2}\left(\mathbb{R}^{n}\right)\right]_{-\theta_{d}}\right)
$$

and that there exists a constant $c>0$ independent of $\psi, h$, and $x_{0}$ such that

$$
\|\psi\|_{\mathcal{C}\left(\bar{I},\left[H^{2}\left(\mathbb{R}^{n}\right), L^{2}\left(\mathbb{R}^{n}\right)\right]_{1 / 2-\theta_{d}}\right)}+\left\|\partial_{t} \psi\right\|_{\mathcal{C}\left(\bar{I},\left[L^{2}\left(\mathbb{R}^{n}\right), H^{-2}\left(\mathbb{R}^{n}\right)\right]_{-\theta_{d}}\right)} \leq c\|h\|_{L^{2}(I)}
$$

holds. This results can be transferred to bounded domains. For that purpose we introduce a smooth cut-off function $\xi \in \mathcal{C}_{c}^{\infty}(\Omega)$ with the property that $\left.\xi\right|_{\Omega_{c}}=1$. Then we introduce the function $\psi_{0}=\xi \psi \in \mathcal{C}\left(\bar{I}, X_{\theta_{d}+1 / 2}\right) \cap \mathcal{C}^{1}\left(\bar{I}, X_{\theta_{d}}\right)$. This function solves the equation

$$
\left\{\begin{aligned}
\partial_{t t} \psi_{0}-\Delta \psi_{0} & =h \delta_{x_{0}}+f & & \text { in } I \times \Omega, \\
\psi_{0} & =0 & & \text { on } I \times \partial \Omega, \\
\left(\psi_{0}, \partial_{t} \psi_{0}\right) & =0 & & \text { in }\{0\} \times \Omega
\end{aligned}\right.
$$

with $f=-\Delta \xi \psi-2 \nabla \xi \cdot \nabla \psi$ in the very weak sense. Additionally we introduce the function $w=\psi_{0}-y$ which is a very weak solution of

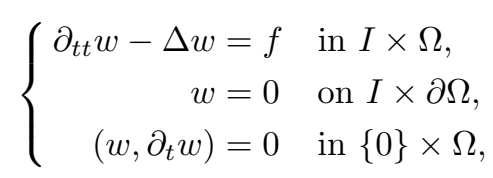

Copyright $@$ ㅇ by SIAM. Unauthorized reproduction of this article is prohibited. 
where $y$ is the very weak solution of $(5.1)$. Since $\psi \in \mathcal{C}\left(\bar{I},\left[H^{2}\left(\mathbb{R}^{n}\right), L^{2}\left(\mathbb{R}^{n}\right)\right]_{1 / 2-\theta_{d}}\right)$ we have

$$
f=-\Delta \xi \psi-2 \nabla \xi \cdot \nabla \psi \in \mathcal{C}\left(\bar{I}, X_{\theta_{d}}\right) .
$$

Therefore, Corollary 4.9 implies that

$$
w \in \mathcal{C}\left(\bar{I}, X_{\theta_{d}+1 / 2}\right) \cap \mathcal{C}^{1}\left(\bar{I}, X_{\theta_{d}}\right) \cap H^{2}\left(I, X_{\theta_{d}-1 / 2}\right)
$$

and

$$
\|w\|_{\mathcal{C}\left(\bar{I}, X_{\theta_{d}+1 / 2}\right)}+\left\|\partial_{t} w\right\|_{\mathcal{C}\left(\bar{I}, X_{\theta_{d}}\right)}+\left\|\partial_{t t} w\right\|_{L^{2}\left(I, X_{\theta_{d}-1 / 2}\right)} \leq c\|f\|_{L^{2}\left(I, X_{\theta_{d}}\right)} .
$$

According to the definition of $w$ we have $y=\psi_{0}-w$, which implies that

$$
y \in \mathcal{C}\left(\bar{I}, X_{\theta_{d}+1 / 2}\right) \cap \mathcal{C}^{1}\left(\bar{I}, X_{\theta_{d}}\right) .
$$

Finally we show the a priori estimate (5.2). We use $y=\psi_{0}-w$, the a priori estimate (5.5) for the $w$-problem, and the definition of $f$ to get

$$
\begin{aligned}
\|y\|_{\mathcal{C}\left(\bar{I}, X_{\theta_{d}+1 / 2}\right)} & \leq\left\|\psi_{0}\right\|_{\mathcal{C}\left(\bar{I}, X_{\theta_{d}+1 / 2}\right)}+\|w\|_{\mathcal{C}\left(\bar{I}, X_{\theta_{d}+1 / 2}\right)} \\
& \leq c\left(\|\xi \psi\|_{\mathcal{C}\left(\bar{I}, X_{\theta_{d}+1 / 2}\right)}+\|\Delta \xi \psi\|_{\mathcal{C}\left(\bar{I}, X_{\theta_{d}+1 / 2}\right)}+\|\nabla \xi \cdot \nabla \psi\|_{\mathcal{C}\left(\bar{I}, X_{\theta_{d}}\right)}\right) .
\end{aligned}
$$

Then we use the estimate

$$
\begin{aligned}
& \|\nabla \xi \cdot \nabla \psi\|_{\mathcal{C}\left(\bar{I}, X_{\theta_{d}}\right)}+\|\Delta \xi \psi\|_{\mathcal{C}\left(\bar{I}, X_{\theta_{d}+1 / 2}\right)}+\|\xi \psi\|_{\mathcal{C}\left(\bar{I}, X_{\theta_{d}+1 / 2}\right)} \\
& \quad \leq c\left(\|\xi\|_{L^{\infty}(\Omega)}+\|\nabla \xi\|_{L^{\infty}(\Omega)}+\|\Delta \xi\|_{L^{\infty}(\Omega)}\right)\|\psi\|_{\mathcal{C}\left(\bar{I},\left[H^{2}\left(\mathbb{R}^{n}\right), L^{2}\left(\mathbb{R}^{n}\right)\right]_{1 / 2-\theta_{d}}\right)},
\end{aligned}
$$

which follows by interpolation of the continuous operators $\psi \mapsto \xi \psi, \psi \mapsto \Delta \xi \psi$ and $\psi \mapsto \nabla \xi \cdot \nabla \psi$. By assumption $\Omega_{c}$ is compact, so there exists a constant $c\left(\Omega_{c}\right)>0$ such that

$$
\|\xi\|_{L^{\infty}(\Omega)}+\|\nabla \xi\|_{L^{\infty}(\Omega)}+\|\Delta \xi\|_{L^{\infty}(\Omega)} \leq c
$$

holds. Then (5.4) implies the assertion. The estimate for $\left\|\partial_{t} y\right\|_{\mathcal{C}\left(\bar{I}, X_{\theta_{d}}\right)}$ can be derived analogously. The estimate

$$
\left\|\partial_{t t} y\right\|_{L^{2}\left(I, X_{\theta_{d}-1 / 2}\right)} \leq c\left(\Omega_{c}\right)\|h\|_{L^{2}(I)}
$$

follows from

$$
\begin{aligned}
\int_{0}^{T}\langle y, v\rangle_{X_{\theta_{d}+1 / 2}, X_{-\theta_{d}-1 / 2}} \partial_{t t} \psi \mathrm{d} t=\int_{0}^{T}\langle y, \Delta v\rangle_{X_{\theta_{d}+1 / 2}, X_{-\theta_{d}-1 / 2}} \psi \mathrm{d} t \\
+(h, \psi)_{L^{2}(I)} v\left(x_{0}\right) \\
\leq c\left(\|y\|_{\mathcal{C}\left(\bar{I}, X_{\theta_{d}+1 / 2}\right)}+\|h\|_{L^{2}(I)}\right)\|v\|_{X_{-\theta_{d}+1 / 2}}\|\psi\|_{L^{2}(I)}
\end{aligned}
$$

for any $\psi \in \mathcal{C}_{c}^{\infty}(I)$ and $v \in X_{-\theta_{d}+1 / 2}$. The equation in (5.6) is proven by setting $\phi=\partial_{t t} \psi v-\Delta v \psi$ in the very weak formulation for $y$.

Remark 5.2. The compactness assumption on $\Omega_{c}$ is essential in the proof of Proposition 5.1. The crucial point is the need for the regularity of the cut-off function $\xi \in \mathcal{C}_{c}^{\infty}(\Omega)$. We demand that $\left.\xi\right|_{\Omega_{c}}=1$. Suppose that $\operatorname{dist}\left(\Omega_{c}, \partial \Omega\right) \rightarrow 0$. Then there would hold

$$
\|\nabla \xi\|_{L^{\infty}(\Omega)}+\|\Delta \xi\|_{L^{\infty}(\Omega)} \rightarrow \infty .
$$

Copyright $@$ by SIAM. Unauthorized reproduction of this article is prohibited. 
This implies

$$
\|y\|_{\mathcal{C}\left(\bar{I}, X_{\theta_{d}+1 / 2}\right)} \leq c\left(\|\nabla \xi\|_{L^{\infty}(\Omega)},\|\Delta \xi\|_{L^{\infty}(\Omega)}\right)\|h\|_{L^{2}(I)} \rightarrow \infty \text { for } \operatorname{dist}\left(\Omega_{c}, \partial \Omega\right) \rightarrow 0
$$

which makes this estimate unusable. Proposition 5.1 also can be proved using other techniques. For instance, in the cases $d=1$ and $d=3$, we can proceed as follows. Let $\psi$ be the solution of (5.3). Further let $z$ be a function satisfying the following equation:

$$
\left\{\begin{aligned}
\partial_{t t} z-\Delta z=0 & \text { in } I \times \Omega \\
z=g & \text { on } I \times \partial \Omega \\
\left(z, \partial_{t} z\right)=0 & \text { in }\{0\} \times \Omega .
\end{aligned}\right.
$$

Problem (5.7) has a unique solution $z \in \mathcal{C}\left(\bar{I},\left[H^{2}(\Omega), L^{2}(\Omega)\right]_{1 / 2-\theta_{d}}\right)$ for Dirichlet data from $g \in O$ with $O=L^{2}(I \times \partial \Omega)$ for $d=3$ and $O=H^{1}(I)^{2}$ for $d=1$ and the a priori estimate

$$
\|z\|_{\mathcal{C}\left(\bar{I},\left[H^{2}(\Omega), L^{2}(\Omega)\right]_{1 / 2-\theta_{d}}\right)} \leq c\|g\|_{O}
$$

holds; see, e.g., [23]. The solution of (5.1) is given by $y=\psi-z$ if $g=\left.\psi\right|_{\partial \Omega}$, where $\psi$ is the solution of the free space problem (5.3). Therefore we can estimate

$$
\begin{aligned}
\|y\|_{\mathcal{C}\left(\bar{I}, X_{\theta_{d}+1 / 2}\right)} & \leq\|\psi\|_{\mathcal{C}\left(\bar{I},\left[H^{2}\left(\mathbb{R}^{n}\right), L^{2}\left(\mathbb{R}^{n}\right)\right]_{1 / 2-\theta_{d}}\right)}+\|z\|_{\mathcal{C}\left(\bar{I},\left[H^{2}(\Omega), L^{2}(\Omega)\right]_{1 / 2-\theta_{d}}\right)} \\
& \leq\|\psi\|_{\mathcal{C}\left(\bar{I},\left[H^{2}\left(\mathbb{R}^{n}\right), L^{2}\left(\mathbb{R}^{n}\right)\right]_{1 / 2-\theta_{d}}\right)}+c\|\psi\|_{O} .
\end{aligned}
$$

In order to establish (5.2) we need to derive an estimate of the following form:

$$
\|\psi\|_{O} \leq c\|h\|_{L^{2}(I)} .
$$

Furthermore, we have to investigate the dependence of $c$ on $\operatorname{dist}\left(\Omega_{c}, \partial \Omega\right)$. In the case $d=1$ it is easy to show by using the explicit solution formula for problem (5.3) that the constant in (5.8) is bounded for $\operatorname{dist}\left(\Omega_{c}, \partial \Omega\right) \rightarrow 0$. In the case $d=3$ the estimate

$$
\|\psi\|_{\mathcal{C}\left(\bar{I}, L^{2}(\partial \Omega)\right)} \leq \frac{c}{\operatorname{dist}\left(\Omega_{c}, \partial \Omega\right)}\|h\|_{L^{2}(I)}
$$

is shown by Lions in [25] by using the explicit solution formula. Estimate (5.9) is sharp in the sense that it can happen that

$$
\|\psi\|_{\mathcal{C}\left(\bar{I}, L^{2}(\partial \Omega)\right)} \rightarrow \infty \text { for } \operatorname{dist}\left(x_{0}, \partial \Omega\right) \rightarrow 0
$$

where $x_{0}$ is the position of the point source. This is shown by the following example. Let $\Omega$ have the following form:

$$
\Omega=\left\{x \in \mathbb{R}^{3}: x_{1}^{2}+x_{2}^{2}<1, \quad-1<x_{3}<0\right\} .
$$

We set $h \equiv 1$ and $x_{0}=(0,0,-\varrho)$ with $\varrho>0$. The solution of $(5.3)$ in this case is given by

$$
\psi(t, x)= \begin{cases}\frac{1}{4 \pi} \frac{1}{\left\|x-x_{0}\right\|_{\mathbb{R}^{3}}} & \text { if } t \geq\left\|x-x_{0}\right\|_{\mathbb{R}^{3}}, \\ 0 & \text { else }\end{cases}
$$

see [25]. Next we calculate $\|\psi(t)\|_{L^{2}\left(\partial \Omega_{1}\right)}$ for 


$$
\partial \Omega_{1}=\left\{x \in \mathbb{R}^{3}: x_{1}^{2}+x_{2}^{2} \leq 1, x_{3}=0\right\} .
$$

This amounts to

$$
\|\psi(t)\|_{L^{2}\left(\partial \Omega_{1}\right)}= \begin{cases}\frac{1}{4 \sqrt{\pi}}\left(\ln \left(t^{2}\right)-\ln \left(\varrho^{2}\right)\right)^{\frac{1}{2}} & \text { if } t \geq \varrho, \\ 0 & \text { else, }\end{cases}
$$

and therefore it holds that

$$
\|\psi\|_{\mathcal{C}\left(\bar{I}, L^{2}(\partial \Omega)\right)} \rightarrow \infty \text { for } \varrho \rightarrow 0 .
$$

In the case $d=2$ the explicit solution formula has a more complicated structure and therefore an estimate of the form (5.8) has not been obtained. Thus, this approach is not applicable.

Next we show that the solution $p$ of (4.4) lies in $\mathcal{C}\left(\Omega_{c}, L^{2}(I)\right)$ for certain classes of data using the previous regularity result for the primal equation.

Theorem 5.3. Let $\left(\phi, p_{1}, p_{0}\right) \in L^{1}\left(I, X_{-\theta_{d}-1 / 2}\right) \times X_{-\theta_{d}-1 / 2} \times X_{-\theta_{d}}$. Then the solution $p$ of (4.4) satisfies

$$
p \in \mathcal{C}\left(\Omega_{c}, L^{2}(I)\right) \cap \mathcal{C}\left(\bar{I}, X_{-\theta_{d}}\right) \cap \mathcal{C}^{1}\left(\bar{I}, X_{-\theta_{d}-1 / 2}\right) \cap W^{2,1}\left(I, X_{-\theta_{d}-1}\right)
$$

and there exists a constant $c>0$ such that

$$
\|p\|_{\mathcal{C}\left(\Omega_{c}, L^{2}(I)\right)} \leq c\left(\|\phi\|_{L^{1}\left(I, X_{-\theta_{d}-1 / 2}\right)}+\left\|p_{0}\right\|_{X_{-\theta_{d}}}+\left\|p_{1}\right\|_{X_{-\theta_{d}-1 / 2}}\right)
$$

holds.

Proof. We intend to show $\mathcal{C}\left(\Omega_{c}, L^{2}(I)\right)$ regularity of $p$. For that we choose the following approximating sequences:

- $\left\{\phi_{n}\right\}_{n \in \mathbb{N}} \subset \mathcal{C}_{c}^{\infty}(I \times \Omega)$ with $\phi_{n} \rightarrow \phi$ in $L^{1}\left(I, X_{-\theta_{d}-1 / 2}\right)$,

- $\left\{p_{0, n}\right\}_{n \in \mathbb{N}} \subset \mathcal{C}_{c}^{\infty}(\Omega)$ with $p_{0, n} \rightarrow p_{0}$ in $X_{-\theta_{d}}$,

- $\left\{p_{1, n}\right\}_{n \in \mathbb{N}} \subset \mathcal{C}_{c}^{\infty}(\Omega)$ with $p_{1, n} \rightarrow p_{1}$ in $X_{-\theta_{d}-1 / 2}$.

Then we consider the following equation:

$$
\left\{\begin{aligned}
\partial_{t t} p_{n}-\Delta p_{n} & =\phi_{n} & & \text { in } I \times \Omega, \\
p_{n} & =0 & & \text { on } I \times \partial \Omega, \\
\left(p_{n}, \partial_{t} p_{n}\right) & =\left(p_{0, n}, p_{1, n}\right) & & \text { in }\{T\} \times \Omega .
\end{aligned}\right.
$$

For $x_{0} \in \Omega_{c}$ arbitrary, let $\xi_{n}$ be the very weak solution of the following problem:

$$
\left\{\begin{aligned}
\partial_{t t} \xi_{n}-\Delta \xi_{n} & =p_{n}\left(x_{0}, t\right) \delta_{x_{0}} & & \text { in } I \times \Omega, \\
\xi_{n} & =0 & & \text { on } I \times \partial \Omega, \\
\left(\xi_{n}, \partial_{t} \xi_{n}\right) & =0 & & \text { in }\{0\} \times \Omega .
\end{aligned}\right.
$$

Proposition 5.1 implies that

$$
\xi_{n} \in \mathcal{C}\left(\bar{I}, X_{\theta_{d}+1 / 2}\right) \cap \mathcal{C}^{1}\left(\bar{I}, X_{\theta_{d}}\right) \cap H^{2}\left(I, X_{\theta_{d}-1 / 2}\right)
$$

as well as the existence of a constant $c>0$ independent of $x_{0}$ such that

$$
\left\|\xi_{n}\right\|_{\mathcal{C}\left(\bar{I}, X_{\theta_{d}+1 / 2}\right)}+\left\|\partial_{t} \xi_{n}\right\|_{\mathcal{C}\left(\bar{I}, X_{\theta_{d}}\right)} \leq c\left\|p_{n}\left(x_{0}, \cdot\right)\right\|_{L^{2}(I)}
$$

Copyright $@$ ( ) by SIAM. Unauthorized reproduction of this article is prohibited. 
holds. Using the very weak formulation (4.5) of (5.12) and (5.13) we can estimate

$$
\begin{gathered}
\left\|p_{n}\left(x_{0}, \cdot\right)\right\|_{L^{2}(I)}^{2}=\int_{0}^{T}\left\langle\xi_{n}, \phi_{n}\right\rangle_{V^{*}, V} \mathrm{~d} t-\left\langle\xi_{n}(T), p_{1, n}\right\rangle_{V^{*}, V}+\left\langle\partial_{t} \xi_{n}(T), p_{0, n}\right\rangle_{V^{2 *}, V^{2}} \\
=\int_{0}^{T}\left\langle\xi_{n}, \phi_{n}\right\rangle_{X_{\theta_{d}+1 / 2}, X_{-\theta_{d}-1 / 2}} \mathrm{~d} t-\left\langle\xi_{n}(T), p_{1, n}\right\rangle_{X_{\theta_{d}+1 / 2}, X_{-\theta_{d}-1 / 2}} \\
+\left\langle\partial_{t} \xi_{n}(T), p_{0, n}\right\rangle_{X_{\theta_{d}}, X_{-\theta_{d}}} \\
\leq\left\|\xi_{n}\right\|_{\mathcal{C}\left(\bar{I}, X_{\theta_{d}+1 / 2}\right)}\left\|\phi_{n}\right\|_{L^{1}\left(I, X_{-\theta_{d}-1 / 2}\right)}+\left\|\xi_{n}(T)\right\|_{X_{\theta_{d}+1 / 2}}\left\|p_{1, n}\right\|_{X_{-\theta_{d}-1 / 2}} \\
+\left\|\partial_{t} \xi_{n}(T)\right\|_{X_{\theta_{d}}}\left\|p_{0, n}\right\|_{X_{-\theta_{d}}} \\
\leq c\left\|p_{n}\left(x_{0}, \cdot\right)\right\|_{L^{2}(I)}\left(\left\|\phi_{n}\right\|_{L^{1}\left(I, X_{-\theta_{d}-1 / 2}\right)}+\left\|p_{0, n}\right\|_{X_{\theta_{d}-1 / 2}}+\left\|p_{1, n}\right\|_{X_{-\theta_{d}}}\right) .
\end{gathered}
$$

Since $x_{0} \in \Omega_{c}$ was arbitrary and the constant $c$ in the last estimate does not depend on $x_{0}$ according to Proposition 5.1 we get

$$
\left\|p_{n}\right\|_{\mathcal{C}\left(\Omega_{c}, L^{2}(I)\right)} \leq c\left(\left\|\phi_{n}\right\|_{L^{1}\left(I, X_{-\theta_{d}-1 / 2}\right)}+\left\|p_{0, n}\right\|_{X_{-\theta_{d}}}+\left\|p_{1, n}\right\|_{X_{-\theta_{d}-1 / 2}}\right) .
$$

The inequality (5.14) and linearity of (5.11) imply that $\left\{p_{n}\right\}_{n \in \mathbb{N}}$ is a Cauchy sequence in $\mathcal{C}_{0}\left(\Omega, L^{2}(I)\right)$. So there exists a $\tilde{p} \in \mathcal{C}\left(\Omega_{c}, L^{2}(I)\right)$ with $p_{n} \rightarrow \tilde{p}$ in $\mathcal{C}\left(\Omega_{c}, L^{2}(I)\right)$. Finally we have to show that $\tilde{p}$ is a solution of the adjoint wave equation (4.4). We know from Corollary 4.9 and Remark 4.10 that

$$
\begin{aligned}
& \left\|p_{n}\right\|_{\mathcal{C}\left(\bar{I}, X_{-\theta_{d}}\right)}+\left\|\partial_{t} p_{n}\right\|_{\mathcal{C}\left(\bar{I}, X_{-\theta_{d}-1 / 2}\right)}+\left\|\partial_{t t} p_{n}\right\|_{L^{1}\left(\bar{I}, X_{-\theta_{d}-1}\right)} \\
& \quad \leq c\left(\left\|\phi_{n}\right\|_{L^{1}\left(I, X_{-\theta_{d}-1 / 2}\right)}+\left\|p_{0, n}\right\|_{X_{-\theta_{d}}}+\left\|p_{1, n}\right\|_{X_{-\theta_{d}-1 / 2}}\right)
\end{aligned}
$$

holds, which implies that $\left\{p_{n}\right\}_{n \in \mathbb{N}}$ is also a Cauchy sequence in $\mathcal{C}\left(\bar{I}, X_{-\theta_{d}}\right) \cap$ $\mathcal{C}^{1}\left(\bar{I}, X_{-\theta_{d}-1 / 2}\right) \cap W^{2,1}\left(I, X_{-\theta_{d}-1}\right)$. Therefore there holds

$$
\tilde{p} \in \mathcal{C}\left(\bar{I}, X_{-\theta_{d}}\right) \cap \mathcal{C}^{1}\left(\bar{I}, X_{-\theta_{d}-1 / 2}\right) \cap W^{2,1}\left(I, X_{-\theta_{d}-1}\right) .
$$

Then we pass to the limit in

$$
\begin{aligned}
& \int_{0}^{T}\left(p_{n}, f\right)_{H} \mathrm{~d} t+\left(p_{n}(0), y_{1}\right)_{H}-\left(\partial_{t} p_{n}(0), y_{0}\right)_{H} \\
& \quad=\int_{0}^{T}\left(\phi_{n}, y\right)_{H} \mathrm{~d} t+\left(p_{0, n}, \partial_{t} y(T)\right)_{H}-\left(p_{1, n}, y(T)\right)_{H}
\end{aligned}
$$

for all $\left(f, y_{0}, y_{1}\right) \in L^{1}(I, V) \times V^{2} \times V$, where $y \in \mathcal{C}\left(\bar{I}, V^{2}\right) \cap \mathcal{C}^{1}(\bar{I}, V)$ is the solution of (4.1) for the data $\left(f, y_{0}, y_{1}\right)$. We see that $\tilde{p}$ satisfies the very weak formulation (4.5) of (4.4). Consequently $\tilde{p}$ is a very weak solution of (4.4).

Remark 5.4. The results of Proposition 5.1 and Theorem 5.3 can be interpreted as a hidden regularity result for the linear wave equation; cf. [23].

In order to establish higher regularity of the velocity $\partial_{t} y$ we need the following lemma.

Lemma 5.5. Let $\phi \in W_{0}^{1,1}\left(I, X_{-\theta_{d}}\right)$ and consider the following equation:

$$
\left\{\begin{aligned}
\partial_{t t} p-\Delta p & =\partial_{t} \phi & & \text { in } I \times \Omega, \\
p & =0 & & \text { on } I \times \partial \Omega, \\
\left(p, \partial_{t} p\right) & =0 & & \text { in }\{0\} \times \Omega .
\end{aligned}\right.
$$

Copyright (C) by SIAM. Unauthorized reproduction of this article is prohibited. 
Then there exists a constant $c\left(\Omega_{c}\right)>0$ such that

$$
\|p\|_{\mathcal{C}\left(\Omega_{c}, L^{2}(I)\right)} \leq c\|\phi\|_{L^{1}\left(I, X_{-\theta_{d}}\right)}
$$

holds.

Proof. Since $\partial_{t} \phi \in L^{1}\left(I, X_{-\theta_{d}}\right)$, Corollary 4.9, Remark 4.10, and Theorem 5.3 guarantee the existence of a unique solution $p$ of (5.15) with

$$
p \in \mathcal{C}\left(\bar{I}, X_{-\theta_{d}+1 / 2}\right) \cap \mathcal{C}^{1}\left(\bar{I}, X_{-\theta_{d}}\right) \cap W^{2,1}\left(I, X_{-\theta_{d}-1 / 2}\right) \cap \mathcal{C}\left(\Omega_{c}, L^{2}(I)\right) .
$$

Next choose any $x_{0} \in \Omega_{c}$. Then let $\xi \in \mathcal{C}\left(\bar{I}, X_{\theta_{d}+1 / 2}\right) \cap \mathcal{C}^{1}\left(\bar{I}, X_{\theta_{d}}\right)$ be the very weak solution of (5.1) with $h=p\left(x_{0}, \cdot\right) \delta_{x_{0}}$. Then we proceed similarly as in the proof of Theorem 5.3 using integration by parts (cf. Lemma 4.4):

$$
\begin{aligned}
\left\|p\left(x_{0}\right)\right\|_{L^{2}(I)}^{2} & =\int_{0}^{T}\left\langle\xi, \partial_{t} \phi\right\rangle_{X_{\theta_{d}}, X_{-\theta_{d}}} \mathrm{~d} t \\
& =-\int_{0}^{T}\left\langle\partial_{t} \xi, \phi\right\rangle_{X_{\theta_{d}}, X_{-\theta_{d}}} \mathrm{~d} t \\
& \leq\left\|\partial_{t} \xi\right\|_{\mathcal{C}\left(\bar{I}, X_{\theta_{d}}\right)}\|\phi\|_{L^{1}\left(I, X_{-\theta_{d}}\right)} \\
& \leq c\left(\Omega_{c}\right)\left\|p\left(x_{0}\right)\right\|_{L^{2}(I)}\|\phi\|_{L^{1}\left(I, X_{-\theta_{d}}\right)} .
\end{aligned}
$$

This finishes the proof.

The following proposition establishes higher regularity of the state variable for sources which are smoother in time, in particular from $\mathcal{M}\left(\Omega_{c}, H^{1}(I)\right)$. These results will be extended to $\mathcal{M}\left(\Omega_{c}, L^{2}(I)\right)$ in a combined density and duality argument in Theorem 5.7.

Proposition 5.6. Let $\left(f, y_{0}, y_{1}\right) \in \mathcal{M}\left(\Omega_{c}, H^{1}(I)\right) \times X_{\theta_{d}+1 / 2} \times X_{\theta_{d}}$. Then the solution $y$ of (4.1) has the following regularity properties:

$$
y \in \mathcal{C}\left(\bar{I}, X_{\theta_{d}+1 / 2}\right) \cap \mathcal{C}^{1}\left(\bar{I}, X_{\theta_{d}}\right) \cap \mathcal{C}^{2}\left(\bar{I}, X_{\theta_{d}-1 / 2}\right) .
$$

Proof. First we remark that

$$
\mathcal{M}\left(\Omega_{c}, H^{1}(I)\right) \subset H^{1}\left(I, X_{-d / 4-\varepsilon}\right) \subset H^{1}\left(I, X_{\theta_{d}-1 / 2}\right)
$$

for $\varepsilon>0$ small enough. Indeed, let $u \in \mathcal{M}\left(\Omega_{c}, H^{1}(I)\right)$ and let $u^{\prime} \in L^{\infty}\left(\left(\Omega_{c},|u|\right), H^{1}(I)\right)$ be its Radon-Nikodym derivative. Then the mapping $t \mapsto u^{\prime}(\cdot, t)|u|$ is an element of $H^{1}\left(I, X_{-d / 4-\varepsilon}\right)$. Then we approximate the data by smooth functions, i.e.,

- $\left\{y_{0, n}\right\} \subset \mathcal{C}_{c}^{\infty}(\Omega), y_{0, n} \rightarrow y_{0}$ in $X_{\theta_{d}+1 / 2}$

- $\left\{y_{1, n}\right\} \subset \mathcal{C}_{c}^{\infty}(\Omega), y_{1, n} \rightarrow y_{0}$ in $X_{\theta_{d}}$

- $\left\{f_{n}\right\} \subset \mathcal{C}^{\infty}\left(\bar{I}, \mathcal{C}_{c}^{\infty}(\Omega)\right), f_{n} \rightarrow f$ in $H^{1}\left(I, X_{\theta_{d}-1 / 2}\right)$.

For the smooth versions of the data we consider the equation

$$
\left\{\begin{aligned}
\partial_{t t} y_{n}-\Delta y_{n} & =f_{n} & & \text { in } I \times \Omega \\
y_{n} & =0 & & \text { on } I \times \partial \Omega \\
\left(y_{n}, \partial_{t} y_{n}\right) & =\left(y_{0, n}, y_{1, n}\right) & & \text { in }\{0\} \times \Omega
\end{aligned}\right.
$$

as well as

$$
\left\{\begin{aligned}
\partial_{t t} \tilde{y}_{n}-\Delta \tilde{y}_{n} & =\partial_{t} f_{n} & & \text { in } I \times \Omega, \\
\tilde{y}_{n} & =0 & & \text { on } I \times \partial \Omega, \\
\left(\tilde{y}_{n}, \partial_{t} \tilde{y}_{n}\right) & =\left(y_{1, n}, f_{n}(0)+\Delta y_{0, n}\right) & & \text { in }\{0\} \times \Omega .
\end{aligned}\right.
$$

Copyright (c) by SIAM. Unauthorized reproduction of this article is prohibited. 
It is easy to see that $\partial_{t} y_{n}=\tilde{y}_{n}$. According to Corollary 4.9 the following estimate holds true:

$$
\begin{aligned}
& \left\|\partial_{t} y_{n}\right\|_{\mathcal{C}\left(\bar{I}, X_{\theta_{d}}\right)}+\left\|\partial_{t t} y_{n}\right\|_{\mathcal{C}\left(\bar{I}, X_{\theta_{d}-1 / 2}\right)} \\
& \quad \leq c \cdot\left(\left\|y_{0, n}\right\|_{X_{\theta_{d}+1 / 2}}+\left\|y_{1, n}\right\|_{X_{\theta_{d}}}+\left\|f_{n}\right\|_{H^{1}\left(I, X_{\theta_{d}-1 / 2}\right)}\right) .
\end{aligned}
$$

Then we use the strong formulation of the wave equation and the last estimate in order to get

$$
\begin{aligned}
\left\|y_{n}(t)\right\|_{X_{\theta_{d}+1 / 2}} & =\left\|A^{\theta_{d}-1 / 2} A y_{n}(t)\right\|_{H}=\left\|\left(A^{\theta_{d}-1 / 2}\left(f_{n}-\partial_{t t} y_{n}\right)\right)(t)\right\|_{H} \\
& \leq c\left(\left\|y_{0, n}\right\|_{X_{\theta_{d}+1 / 2}}+\left\|y_{1, n}\right\|_{X_{\theta_{d}}}+\left\|f_{n}\right\|_{H^{1}\left(I, X_{\theta_{d}-1 / 2}\right)}\right)
\end{aligned}
$$

for any $t \in \bar{I}$. The last estimates show that $\left\{y_{n}\right\}$ is a Cauchy sequence in

$$
\mathcal{C}\left(\bar{I}, X_{\theta_{d}+1 / 2}\right) \cap \mathcal{C}^{1}\left(\bar{I}, X_{\theta_{d}}\right) \cap \mathcal{C}^{2}\left(\bar{I}, X_{\theta_{d}-1 / 2}\right) .
$$

A standard limiting argument involving the very weak formulation yields the assertion.

Now we have introduced all preparatory results in order to prove higher regularity of the state.

THEOREM 5.7. Let $\left(u, y_{0}, y_{1}\right) \in \mathcal{M}\left(\Omega_{c}, L^{2}(I)\right) \times X_{\theta_{d}+1 / 2} \times X_{\theta_{d}}$ and $y$ be the very weak solution of the state equation (1.1). Then there holds

$$
y \in \mathcal{C}\left(\bar{I}, X_{\theta_{d}+1 / 2}\right) \cap \mathcal{C}^{1}\left(I, X_{\theta_{d}}\right) \cap H^{2}\left(I, X_{\theta_{d}-1 / 2}\right) .
$$

Furthermore there exists a constant $c>0$ such that

$$
\begin{gathered}
\|y\|_{\mathcal{C}\left(\bar{I}, X_{\theta_{d}+1 / 2}\right)}+\left\|\partial_{t} y\right\|_{\mathcal{C}\left(\bar{I}, X_{\theta_{d}}\right)}+\left\|\partial_{t t} y\right\|_{L^{2}\left(I, X_{\theta_{d}-1 / 2}\right)} \\
\leq c\left(\|u\|_{\mathcal{M}\left(\Omega_{c}, L^{2}(I)\right)}+\left\|y_{0}\right\|_{X_{\theta_{d}+1 / 2}}+\left\|y_{1}\right\|_{X_{\theta_{d}}}\right)
\end{gathered}
$$

holds.

Proof. Corollary 4.11 implies the existence of a unique solution $y$ of (1.1) which satisfies

$$
y \in \mathcal{C}\left(\bar{I}, X_{-(d-2) / 4-\varepsilon}\right) \cap \mathcal{C}^{1}\left(\bar{I}, X_{-d / 4-\varepsilon}\right) \cap H^{2}\left(I, X_{-(d+2) / 4-\varepsilon}\right), \quad 1 / 4>\varepsilon>0 .
$$

But the regularity of $y$ can be essentially improved in the following way. First of all we consider the case with homogeneous initial data $y_{0}=y_{1}=0$. Proposition 2.1 implies the existence of a sequence $\left\{u_{n}\right\}_{n \in \mathbb{N}} \subset \mathcal{M}\left(\Omega_{c}, H^{1}(I)\right)$ with

$$
\left\|u-u_{n}\right\|_{\mathcal{M}\left(\Omega_{c}, L^{2}(I)\right)} \rightarrow 0 \text { for } n \rightarrow \infty .
$$

Now we consider the problem

$$
\left\{\begin{aligned}
\partial_{t t} y_{n}-\Delta y_{n}=u_{n} & & \text { in } I \times \Omega, \\
y_{n}=0 & & \text { on } I \times \partial \Omega, \\
\left(y_{n}, \partial_{t} y_{n}\right)=0 & & \text { in }\{0\} \times \Omega,
\end{aligned}\right.
$$

which has a solution unique solution $y_{n}$ with

$$
y_{n} \in \mathcal{C}\left(\bar{I}, X_{\theta_{d}+1 / 2}\right) \cap \mathcal{C}^{1}\left(I, X_{\theta_{d}}\right) \cap \mathcal{C}^{2}\left(I, X_{\theta_{d}-1 / 2}\right)
$$

Copyright (c) by SIAM. Unauthorized reproduction of this article is prohibited. 
according to Proposition 5.6. Next we prove the estimate

$$
\left\|y_{n}\right\|_{L^{\infty}\left(I, X_{\theta_{d}+1 / 2}\right)} \leq c\left\|u_{n}\right\|_{\mathcal{M}\left(\Omega_{c}, L^{2}(I)\right)}
$$

using the very weak formulation of (5.18) in the form

$$
\int_{0}^{T}\left\langle y_{n}, \phi\right\rangle_{X_{\theta_{d}+1 / 2}, X_{-\theta_{d}-1 / 2}} \mathrm{~d} t=\left\langle u_{n}, p\right\rangle_{\mathcal{M}\left(\Omega_{c}, L^{2}(I)\right), \mathcal{C}\left(\Omega_{c}, L^{2}(I)\right)},
$$

where $p$ is the solution of

$$
\left\{\begin{aligned}
\partial_{t t} p-\Delta p=\phi & \text { in } I \times \Omega, \\
p=0 & \text { on } I \times \partial \Omega, \\
\left(p, \partial_{t} p\right)=0 & \text { in }\{T\} \times \Omega
\end{aligned}\right.
$$

with $\phi \in L^{1}\left(I, X_{-\theta_{d}-1 / 2}\right)$. According to Theorem 5.3 this equation has a unique solution $p \in \mathcal{C}\left(\Omega_{c}, L^{2}(I)\right)$ which fulfills the following estimate:

$$
\|p\|_{\mathcal{C}\left(\Omega_{c}, L^{2}(I)\right)} \leq c\|\phi\|_{L^{1}\left(I, X_{-\theta_{d^{-1} / 2}}\right)} .
$$

Therefore we can estimate in the following manner:

$$
\begin{aligned}
\int_{0}^{T}\left\langle y_{n}, \phi\right\rangle_{X_{\theta_{d}+1 / 2}, X_{-\theta_{d}-1 / 2}} \mathrm{~d} t & =\left\langle u_{n}, p\right\rangle_{\mathcal{M}\left(\Omega_{c}, L^{2}(I)\right), \mathcal{C}\left(\Omega_{c}, L^{2}(I)\right)} \\
& \leq\left\|u_{n}\right\|_{\mathcal{M}\left(\Omega_{c}, L^{2}(I)\right)}\|p\|_{\mathcal{C}\left(\Omega_{c}, L^{2}(I)\right)} \\
& \leq c\left\|u_{n}\right\|_{\mathcal{M}\left(\Omega_{c}, L^{2}(I)\right)}\|\phi\|_{L^{1}\left(I, X_{-\theta_{d}-1 / 2}\right)} .
\end{aligned}
$$

This implies that

$$
\left\|y_{n}\right\|_{L^{\infty}\left(I, X_{\theta_{d}+1 / 2}\right)} \leq c\left\|u_{n}\right\|_{\mathcal{M}\left(\Omega_{c}, L^{2}(I)\right)}
$$

holds. We proceed with an estimate of the form

$$
\left\|\partial_{t} y_{n}\right\|_{L^{\infty}\left(I, X_{\theta_{d}}\right)} \leq c\left\|u_{n}\right\|_{\mathcal{M}\left(\Omega_{c}, L^{2}(I)\right)}
$$

using the very weak formulation of (5.18) involving the solution $p$ of

$$
\left\{\begin{array}{rlrl}
\partial_{t t} p-\Delta p & =\partial_{t} \phi & & \text { in } I \times \Omega, \\
p=0 & & \text { on } I \times \partial \Omega, \\
\left(p, \partial_{t} p\right) & =0 & & \text { in }\{T\} \times \Omega
\end{array}\right.
$$

with $\phi \in W_{0}^{1,1}\left(I, X_{-\theta_{d}}\right)$. According to Theorem 5.3 this equation has a unique solution $p \in \mathcal{C}\left(\Omega_{c}, L^{2}(I)\right)$ and the estimate

$$
\|p\|_{\mathcal{C}\left(\Omega_{c}, L^{2}(I)\right)} \leq c\|\phi\|_{L^{1}\left(I, X_{-\theta_{d}}\right)}
$$

holds according to Lemma 5.5. We choose any $\phi \in W_{0}^{1,1}(I, V)$ and have after integration by parts in time (cf. Lemma 4.4) that

$$
\begin{aligned}
\int_{0}^{T}\left\langle\partial_{t} y_{n}, \phi\right\rangle_{X_{\theta_{d}}, X_{-\theta_{d}}} \mathrm{~d} t & =-\int_{0}^{T}\left\langle y_{n}, \partial_{t} \phi\right\rangle_{X_{\theta_{d}+1 / 2}, X_{-\theta_{d}-1 / 2}} \mathrm{~d} t \\
& =-\left\langle u_{n}, p\right\rangle_{\mathcal{M}\left(\Omega_{c}, L^{2}(I)\right), \mathcal{C}\left(\Omega_{c}, L^{2}(I)\right)} \\
& \leq\left\|u_{n}\right\|_{\mathcal{M}\left(\Omega_{c}, L^{2}(I)\right)}\|p\|_{\mathcal{C}\left(\Omega_{c}, L^{2}(I)\right)} \\
& \left.\leq c\left\|u_{n}\right\|_{\mathcal{M}\left(\Omega_{c}, L^{2}(I)\right)}\|\phi\|_{L^{1}\left(I, X_{-\theta_{d}}\right.}\right)
\end{aligned}
$$

Copyright (c) by SIAM. Unauthorized reproduction of this article is prohibited. 
The density of $W_{0}^{1,1}\left(I, X_{-\theta_{d}}\right)$ in $L^{1}\left(I, X_{-\theta_{d}}\right)$ yields the desired estimate

$$
\left\|\partial_{t} y_{n}\right\|_{L^{\infty}\left(I, X_{\theta_{d}}\right)} \leq c\left\|u_{n}\right\|_{\mathcal{M}\left(\Omega_{c}, L^{2}(I)\right)} .
$$

The estimate

$$
\left\|\partial_{t t} y_{n}\right\|_{L^{2}\left(I, X_{\theta_{d}-1 / 2}\right)} \leq c\left\|u_{n}\right\|_{\mathcal{M}\left(\Omega_{c}, L^{2}(I)\right)}
$$

follows from

$$
\begin{aligned}
\int_{0}^{T}\left\langle y_{n}, v\right\rangle_{X_{\theta_{d}+1 / 2}, X_{-\theta_{d}-1 / 2}} \partial_{t t} \psi \mathrm{d} t= & \int_{0}^{T}\left\langle y_{n}, \Delta v\right\rangle_{X_{\theta_{d}+1 / 2}, X_{-\theta_{d}-1 / 2}} \psi \mathrm{d} t \\
& +\left\langle u_{n}, v \psi\right\rangle_{\mathcal{M}\left(\Omega_{c}, L^{2}(I)\right), \mathcal{C}\left(\Omega_{c}, L^{2}(I)\right)}
\end{aligned}
$$

for any $\psi \in \mathcal{C}_{c}^{\infty}(I)$ and $v \in X_{-\theta_{d}+1 / 2}$ in the same manner as in the proof of Proposition 5.1. Now we take any $n, m \in \mathbb{N}$ and use the linearity of the state equation to obtain the following estimate:

$$
\begin{aligned}
\left\|y_{n}-y_{m}\right\|_{L^{\infty}\left(I, X_{\theta_{d}+1 / 2}\right)} & +\left\|\partial_{t}\left(y_{n}-y_{m}\right)\right\|_{L^{\infty}\left(I, X_{\theta_{d}}\right)} \\
& +\left\|\partial_{t t}\left(y_{n}-y_{m}\right)\right\|_{L^{2}\left(I, X_{\theta_{d}-1 / 2}\right)} \leq c\left\|u_{n}-u_{m}\right\|_{\mathcal{M}\left(\Omega_{c}, L^{2}(I)\right)} .
\end{aligned}
$$

Hence (5.17) implies that $\left\{y_{n}\right\}_{n \in \mathbb{N}}$ is a Cauchy sequence in

$$
\mathcal{C}\left(\bar{I}, X_{\theta_{d}+1 / 2}\right) \cap \mathcal{C}\left(\bar{I}, X_{\theta_{d}}\right) \cap H^{2}\left(I, X_{\theta_{d}-1 / 2}\right) .
$$

Therefore there exists an element $y \in \mathcal{C}\left(\bar{I}, X_{\theta_{d}+1 / 2}\right) \cap \mathcal{C}^{1}\left(\bar{I}, X_{\theta_{d}}\right) \cap H^{2}\left(I, X_{\theta_{d}-1 / 2}\right)$ with $\left(y_{n}, \partial_{t} y_{n}, \partial_{t t} y_{n}\right) \rightarrow\left(y, \partial_{t} y, \partial_{t t} y\right)$. We pass to the limit in the very weak formulation of (5.18) and see that $y$ fulfills the very weak formulation of the state equation. Therefore $y$ is a very weak solution of the state equation. Due to the linearity of the state equation the case with inhomogeneous initial data can be treated separately using Corollary 4.9.

6. Existence of optimal controls. In this section we prove the well-posedness of $(\mathcal{P})$, employing the regularity results of the last section. Throughout the remainder of this paper we assume that $\left(y_{0}, y_{1}\right) \in X_{\theta_{d}+1 / 2} \times X_{\theta_{d}}$. The control-to-state mapping is denoted by

$$
S: \mathcal{M}\left(\Omega_{c}, L^{2}(I)\right) \rightarrow L^{2}(I \times \Omega) \times H \times V^{*}, u \mapsto\left(y, y(T, \cdot), \partial_{t} y(T, \cdot)\right),
$$

where $y$ is the very weak solution of (1.1) for a control $u$. According to Theorem 5.7, it is a bounded affine linear operator. Furthermore we specify the data tracking functional

$$
J\left(y_{1}, y_{2}, y_{3}\right)=\frac{1}{2}\left\{\nu_{1}\left\|y_{1}-z_{1}\right\|_{L^{2}(I \times \Omega)}^{2}+\nu_{2}\left\|y_{2}-z_{2}\right\|_{H}^{2}+\nu_{3}\left\|y_{3}-z_{3}\right\|_{V^{*}}^{2}\right\}
$$

for $\left(y_{1}, y_{2}, y_{3}\right) \in L^{2}(I \times \Omega) \times H \times V^{*},\left(z_{1}, z_{2}, z_{3}\right) \in L^{2}(I \times \Omega) \times H \times V^{*}$, and $\nu_{i} \geq 0$. Using the operator $S$ we introduce the reduced cost functional

$$
j(u)=J(S u)+\alpha\|u\|_{\mathcal{M}\left(\Omega_{c}, L^{2}(I)\right)} .
$$

Next we proof weak continuity properties of $S$.

Lemma 6.1. Let $\left\{u_{n}\right\}_{n \in \mathbb{N}} \subset \mathcal{M}\left(\Omega_{c}, L^{2}(I)\right)$ and $u \in \mathcal{M}\left(\Omega_{c}, L^{2}(I)\right)$ with $u_{n} \rightarrow^{*} u$ in $\mathcal{M}\left(\Omega, L^{2}(I)\right)$. Then there holds $S u_{n} \rightarrow^{*} S u$ in $L^{\infty}(I, H) \times H \times V^{*}$ for $d=1,2,3$ and additionally $S u_{n} \rightarrow S u$ in $L^{2}(I \times \Omega) \times H \times V^{*}$ for $d=1,2$. 
Proof. Let $y_{n}$ be the solution of (1.1) for the control $u_{n}$. From the a priori estimate (5.16) it follows that there exist a subsequence of $\left\{y_{n}\right\}_{n \in \mathbb{N}}$ (denoted with the same index $n$ ) and elements

$$
(y, \hat{y}, \tilde{y}) \in L^{\infty}(I, H) \cap H^{1}\left(I, V^{*}\right) \cap H^{2}\left(I, V^{2 *}\right) \times H \times V^{*}
$$

such that

$$
\left(y_{n}, y_{n}(T), \partial_{t} y_{n}(T)\right) \rightarrow^{*}(y, \hat{y}, \tilde{y}) \quad \text { in } L^{\infty}(I, H) \cap H^{1}\left(I, V^{*}\right) \cap H^{2}\left(I, V^{2 *}\right) \times H \times V^{*}
$$

holds. Due to the weak to weak continuity of the time-point evaluation operator

$$
E: H^{1}\left(I, V^{*}\right) \cap H^{2}\left(I, V^{2 *}\right) \rightarrow V^{*} \times V^{2 *}, y \mapsto\left(y(T), \partial_{t} y(T)\right)
$$

there holds $\hat{y}=y(T)$ and $\tilde{y}=\partial_{t} y(T)$. By passing to the limit in the very weak formulation (4.5) of the equation for $y_{n}$ we obtain that $y$ fulfills

$$
\begin{aligned}
& \int_{0}^{T}(y, \phi)_{H} \mathrm{~d} t-\left(y(T), p_{1}\right)_{H}+\left\langle\partial_{t} y(T), p_{0}\right\rangle_{V^{*}, V} \\
& \quad=\langle u, p\rangle_{\mathcal{M}\left(\Omega_{c}, L^{2}(I)\right), \mathcal{C}\left(\Omega_{c}, L^{2}(I)\right)}-\left(y_{0}, \partial_{t} p(0)\right)_{H}+\left\langle y_{1}, p(0)\right\rangle_{V^{*}, V}
\end{aligned}
$$

where $p \in \mathcal{C}(\bar{I}, V) \cap \mathcal{C}^{1}(\bar{I}, H) \cap \mathcal{C}\left(\Omega_{c}, L^{2}(I)\right)$ is the solution of (4.4) for any $\left(\phi, p_{1}, p_{0}\right) \in$ $L^{1}(I, H) \times H \times V$. Overall this means that $y$ is a very weak solution of the state equation for the control $u$. From (5.16) we conclude that $\left(y_{n}, y_{n}(T), \partial_{t} y_{n}(T)\right)$ is bounded in

$$
\left(\mathcal{C}\left(\bar{I}, X_{\theta_{d}+1 / 2}\right) \cap \mathcal{C}^{1}\left(\bar{I}, X_{\theta_{d}}\right)\right) \times X_{\theta_{d}+1 / 2} \times X_{\theta_{d}} .
$$

For $d=1,2$ the embedding

$$
\left(\mathcal{C}\left(\bar{I}, X_{\theta_{d}+1 / 2}\right) \cap \mathcal{C}^{1}\left(\bar{I}, X_{\theta_{d}}\right)\right) \times X_{\theta_{d}+1 / 2} \times X_{\theta_{d}} \hookrightarrow L^{2}(I \times \Omega) \times H \times V^{*}
$$

is compact according to the Aubin-Lions lemma [27, Chapter 3, Proposition 1.3]. This implies strong convergence of $\left(y_{n}, y_{n}(T), \partial_{t} y_{n}(T)\right)$ in $L^{2}(I \times \Omega) \times H \times V^{*}$. The uniqueness of $y$ implies the convergence of the whole sequence.

Now we are ready to prove the well-posedness of problem $(\mathcal{P})$ by classical arguments.

Proposition 6.2. Problem $(\mathcal{P})$ has a solution $\bar{u} \in \mathcal{M}\left(\Omega_{c}, L^{2}(I)\right)$ which is unique for $\nu_{1}>0$.

Proof. Since $j$ is bounded from below there exists a sequence

$$
\left\{u_{n}\right\}_{n \in \mathbb{N}} \in \mathcal{M}\left(\Omega_{c}, L^{2}(I)\right)
$$

with $j\left(u_{n}\right) \rightarrow \inf _{u \in \mathcal{M}\left(\Omega_{c}, L^{2}(I)\right)} j(u)=\bar{j}$. For all $n \in \mathbb{N}$ large enough it holds that

$$
\alpha\left\|u_{n}\right\|_{\mathcal{M}\left(\Omega_{c}, L^{2}(I)\right)} \leq j\left(u_{n}\right) \leq j(0)+1 .
$$

We recall the weak-* sequential precompactness of bounded sets in $\mathcal{M}\left(\Omega_{c}, L^{2}(I)\right)$ from section 2. Consequently there exists a subsequence $\left\{u_{n_{k}}\right\}_{k \in \mathbb{N}}$ and $\bar{u} \in$ $\mathcal{M}\left(\Omega_{c}, L^{2}(I)\right)$ with $u_{n_{k}} \rightarrow^{*} \bar{u}$ in $\mathcal{M}\left(\Omega_{c}, L^{2}(I)\right)$. From Lemma 6.1 we know $S u_{n_{k}} \rightarrow^{*}$ $S \bar{u}$ in $L^{\infty}(I, H) \times H \times V^{*}$. Moreover $J$ is weak lower semicontinuous in $L^{2}(I, H) \times$ $H \times V^{*}$ and $\|\cdot\|_{\mathcal{M}\left(\Omega_{c}, L^{2}(I)\right)}$ is weak-* lower semicontinuous in $\mathcal{M}\left(\Omega_{c}, L^{2}(I)\right)$. So there holds 


$$
\begin{aligned}
\bar{j}=\liminf _{k \rightarrow \infty} j\left(u_{n_{k}}\right) & \geq \liminf _{k \rightarrow \infty} J\left(S u_{n_{k}}\right)+\liminf _{k \rightarrow \infty} \alpha\left\|u_{n_{k}}\right\|_{\mathcal{M}\left(\Omega_{c}, L^{2}(I)\right)} \\
& \geq J(S \bar{u})+\alpha\|\bar{u}\|_{\mathcal{M}\left(\Omega_{c}, L^{2}(I)\right)},
\end{aligned}
$$

which implies that $\bar{u}$ is a minimizer of $j$.

Remark 6.3. Concerning uniqueness of the optimal control, we observe that it cannot be obtained from the control cost $\|u\|_{\mathcal{M}\left(\Omega_{c}, L^{2}(I)\right)}$ since it is not strictly convex. However, since $\left(y_{1}, y_{2}, y_{3}\right) \mapsto J\left(y_{1}, y_{2}, y_{3}\right)$ is strictly convex, uniqueness of the optimal control follows from injectivity of the control-to-state operator $S$. In the case of solely terminal observation $S$ is not injective $\left(\nu_{1}=0\right)$.

So far we assumed the availability of observations on all of $I \times \Omega$. In the case that the observation domain is of the form $\Omega_{o} \times I$ with $\Omega_{o} \subset \Omega$, existence of a solution to $(\mathcal{P})$ remains correct. Uniqueness of the solution is guaranteed if the control-to-state operator $S: \mathcal{M}\left(\Omega_{c}, L^{2}(I)\right) \rightarrow L^{2}\left(\Omega_{o} \times I\right) \times L^{2}\left(\Omega_{o}\right) \times H^{-1}(\Omega)$ is injective. This is related to the Huygens principle and the location of $\Omega_{o}$ relative to $\Omega$.

In the next section we derive optimality conditions for $(\mathcal{P})$.

7. First order optimality conditions. In this section we derive first order optimality conditions. We rely on similar arguments in [20]. Furthermore we use the first order optimality conditions to establish structural properties of the optimal control. Finally we prove improved regularity of the optimal adjoint state and optimal control. First we introduce the predual operator $S^{\prime \star}$ of the linearized control-toobservation operator $S^{\prime}$ which is equal to $S$ for zero initial data. It is given by

$$
S^{\prime \star}: L^{2}(I \times \Omega) \times H \times V \rightarrow \mathcal{C}\left(\Omega_{c}, L^{2}(I)\right), \quad\left(q_{1}, q_{2}, q_{3}\right) \mapsto p,
$$

where $p$ is the solution of (4.4) for $\phi=q_{1}, p_{0}=q_{3}$, and $p_{1}=-q_{2}$ and satisfies

$$
\begin{gathered}
\left(\left(S^{\prime} u\right)_{1}, q_{1}\right)_{L^{2}(I \times \Omega)}+\left(\left(S^{\prime} u\right)_{2}, q_{2}\right)_{H}+\left\langle\left(S^{\prime} u\right)_{3}, q_{3}\right\rangle_{V^{*}, V} \\
=\left\langle u, S^{\prime \star}\left(q_{1}, q_{2}, q_{3}\right)\right\rangle_{\mathcal{M}\left(\Omega_{c}, L^{2}(I)\right), \mathcal{C}\left(\Omega_{c}, L^{2}(I)\right) .}
\end{gathered}
$$

Moreover, it is linear and bounded. It has the mentioned properties according to Theorem 5.3.

THEOREM 7.1. Let $(\bar{u}, \bar{y})$ be a solution of $(\mathcal{P})$. Then there exists a unique adjoint state $\bar{p} \in \mathcal{C}\left(\Omega_{c}, L^{2}(I)\right)$ which solves

$$
\left\{\begin{aligned}
\partial_{t t} \bar{p}-\Delta \bar{p} & =\nu_{1}\left(\bar{y}-z_{1}\right) & & \text { in } I \times \Omega, \\
\bar{p} & =0 & & \text { on } I \times \partial \Omega, \\
\bar{p}(T) & =\nu_{3} A^{-1 / 2}\left(A^{-1 / 2}\right)^{*}\left(\partial_{t} \bar{y}-z_{3}\right) & & \text { in }\{T\} \times \Omega, \\
\partial_{t} \bar{p}(T) & =\nu_{2}\left(z_{2}-\bar{y}\right) & & \text { in }\{T\} \times \Omega
\end{aligned}\right.
$$

and fulfills the subgradient condition

$$
-\bar{p} \in \alpha \partial\|\bar{u}\|_{\mathcal{M}\left(\Omega_{c}, L^{2}(I)\right)}
$$

or equivalently

$$
\alpha\|\bar{u}\|_{\mathcal{M}\left(\Omega_{c}, L^{2}(I)\right)}=\langle\bar{u},-\bar{p}\rangle_{\mathcal{M}\left(\Omega_{c}, L^{2}(I)\right), \mathcal{C}\left(\Omega_{c}, L^{2}(I)\right)}, \quad\|\bar{p}\|_{\mathcal{C}\left(\Omega_{c}, L^{2}(I)\right)} \leq \alpha .
$$

Proof. Problem $(\mathcal{P})$ can be formulated in the form

$$
\min _{u \in \mathcal{M}\left(\Omega_{c}, L^{2}(I)\right)}(J \circ S+F)(u)
$$

Copyright $@$ by SIAM. Unauthorized reproduction of this article is prohibited. 
with $F(u)=\alpha\|u\|_{\mathcal{M}\left(\Omega_{c}, L^{2}(I)\right)}$. The control $\bar{u} \in \mathcal{M}\left(\Omega_{c}, L^{2}(I)\right)$ is a solution of problem $(\mathcal{P})$ if and only if

$$
0 \in \partial(J \circ S+F)(\bar{u})
$$

where $\partial \cdot$ is the subdifferential of a convex function on $\mathcal{M}\left(\Omega_{c}, L^{2}(I)\right)$ equipped with its weak-* topology. Due to the Fréchet differentiability of $J$ the optimality condition can be rewritten as

$$
-S^{\prime \star}\left(J^{\prime}(S \bar{u})\right) \in \partial F(\bar{u})
$$

Next we differentiate $J \circ S$ with respect to $u$ in direction $\delta u \in \mathcal{M}\left(\Omega_{c}, L^{2}(I)\right)$ and obtain the following expression:

$$
\begin{aligned}
& \left\langle J^{\prime}(S u), \delta u\right\rangle_{\mathcal{C}\left(\Omega_{c}, L^{2}(I)\right), \mathcal{M}\left(\Omega_{c}, L^{2}(I)\right)}=\nu_{1}\left((S u)_{1}-z_{1},\left(S^{\prime} \delta u\right)_{1}\right)_{L^{2}(I \times \Omega)} \\
& \quad+\nu_{2}\left((S u)_{2}-z_{2},\left(S^{\prime} \delta u\right)_{2}\right)_{H}+\nu_{3}\left\langle A^{-1 / 2}\left(A^{-1 / 2}\right)^{*}\left((S u)_{3}-z_{3}\right),\left(S^{\prime} u\right)_{3}\right\rangle_{V, V^{*}} .
\end{aligned}
$$

We define

$$
\bar{p}:=S^{\prime \star}\left(\nu_{1}\left((S \bar{u})_{1}-z_{1}\right), \nu_{2}\left((S \bar{u})_{2}-z_{2}\right), \nu_{3} A^{-1 / 2}\left(A^{-1 / 2}\right)^{*}\left((S u)_{3}-z_{3}\right)\right),
$$

which means that $\bar{p}$ is the solution of the adjoint wave equation with right-hand side $\phi=\nu_{1}\left(z_{1}-(S \bar{u})_{1}\right)$, initial displacement $p_{0}=\nu_{3}\left(A^{-1 / 2}\left(A^{-1 / 2}\right)^{*}\left((S \bar{u})_{3}-z_{3}\right)\right)$, and initial velocity $p_{1}=\nu_{2}\left(z_{2}-(S u)_{2}\right)$. Thus (7.3) can be expressed in the following form:

$$
-\bar{p} \in \partial F(\bar{u})
$$

This subgradient condition is equivalent to

$$
F(\bar{u})+F^{\star}(-\bar{p})=\langle\bar{u},-\bar{p}\rangle_{\mathcal{M}\left(\Omega_{c}, L^{2}(I)\right), \mathcal{C}\left(\Omega_{c}, L^{2}(I)\right)}
$$

(e.g., see [14, Proposition 5.1]), where $F^{\star}$ is the convex conjugate of $F$ with respect to the weak-* topology. It is given by

$$
F^{\star}(p)= \begin{cases}0, & \|p\|_{\mathcal{C}\left(\Omega_{c}, L^{2}(I)\right)} \leq \alpha, \\ \infty & \text { else. }\end{cases}
$$

Therefore (7.5) is equivalent to (7.2).

Next we establish structural properties of the optimal control $\bar{u}$.

Proposition 7.2. Let $\bar{u} \in \mathcal{M}\left(\Omega_{c}, L^{2}(I)\right)$ be the solution of $(\mathcal{P}),|\bar{u}|$ its total variation measure, $\bar{u}^{\prime}$ its Radon-Nikodym derivative, and $\bar{p}$ the optimal adjoint state. Then there holds

$$
\begin{aligned}
\bar{u}^{\prime} & =-\frac{1}{\alpha} \bar{p} \quad \text { in } L^{1}\left(\left(\Omega_{c},|\bar{u}|\right), L^{2}(I)\right), \\
\operatorname{supp}|\bar{u}| & \subseteq\left\{x \in \Omega_{c}:\|\bar{p}(x)\|_{L^{2}(I)}=\alpha\right\} .
\end{aligned}
$$

Proof. A proof for these results can be found in [20, Theorem 2.12].

Next we show improved regularity of the optimal adjoint state $\bar{p}$ in space and of the optimal control $\bar{u}$ in time.

Copyright $@$ by SIAM. Unauthorized reproduction of this article is prohibited. 
TheOrem 7.3. Let $\bar{u} \in \mathcal{M}\left(\Omega_{c}, L^{2}(I)\right)$ be the solution of $(\mathcal{P})$ and $\bar{p} \in \mathcal{C}\left(\Omega_{c}, L^{2}(I)\right)$ the corresponding optimal adjoint state. Additionally let $z_{1} \in \mathcal{C}\left(\bar{I}, X_{\theta_{d}+1 / 2}\right), z_{2} \in$ $X_{\theta_{d}+1 / 2}$, and $z_{3} \in X_{\theta_{d}}$. Then the regularity result

$$
\bar{p} \in \mathcal{C}\left(\bar{I}, X_{\theta_{d}+1}\right) \cap \mathcal{C}^{1}\left(\bar{I}, X_{\theta_{d}+1 / 2}\right) \cap \mathcal{C}^{2}\left(\bar{I}, X_{\theta_{d}}\right)
$$

for $d=1,2,3$ and

$$
\bar{u} \in \mathcal{C}^{2-d, 1 / 2-\varepsilon}\left(\bar{I}, \mathcal{M}\left(\Omega_{c}\right)\right)
$$

for $d=1,2$ and any $\varepsilon \in(0,1 / 2]$ holds.

Proof. Due to the assumptions on $z_{1}, z_{2}, z_{3}$ and according to Theorem 5.7 there holds

$$
\begin{aligned}
\phi & =\bar{y}-z_{1} \in \mathcal{C}\left(\bar{I}, X_{\theta_{d}+1 / 2}\right), p_{1}=z_{2}-\bar{y}(T) \in X_{\theta_{d}+1 / 2}, \\
p_{0} & =A^{-1 / 2}\left(A^{-1 / 2}\right)^{*}\left(\partial_{t} \bar{y}(T)-z_{3}\right) \in X_{\theta_{d}+1} \text { for } d=2,3 \\
p_{0} & =A^{-1}\left(\partial_{t} \bar{y}(T)-z_{3}\right) \in V^{2} \text { for } d=1 .
\end{aligned}
$$

So the improved regularity of $\bar{p}$ for $d=1,2$ follows from Remark 4.10 and the fact that the continuity of the right-hand side $\phi$ in time implies the continuity of $\partial_{t t} \bar{p}$ in time. Next we show the improved regularity of $\bar{u}$ for $d=1,2$. We have for any $\varepsilon \in(0,1 / 2]$

$$
\bar{p} \in \mathcal{C}^{1}\left(\bar{I}, X_{1 / 2}\right) \cap \mathcal{C}^{2}\left(\bar{I}, X_{0}\right) \hookrightarrow \mathcal{C}^{1,1 / 2-\varepsilon}\left(\bar{I}, X_{1 / 4+\varepsilon}\right) \hookrightarrow \mathcal{C}^{1,1 / 2-\varepsilon}\left(\bar{I}, \mathcal{C}\left(\Omega_{c}\right)\right) \text { for } d=1
$$

and

$$
\bar{p} \in \mathcal{C}\left(\bar{I}, X_{3 / 4}\right) \cap C^{1}\left(\bar{I}, X_{1 / 4}\right) \hookrightarrow \mathcal{C}^{0,1 / 2-\varepsilon}\left(\bar{I}, X_{1 / 2+\varepsilon}\right) \hookrightarrow \mathcal{C}^{0,1 / 2-\varepsilon}\left(\bar{I}, \mathcal{C}\left(\Omega_{c}\right)\right) \text { for } d=2 .
$$

Details concerning this embedding of vector-valued spaces can be found in [1, Theorem 5.2]. First we consider the case $d=2$. We recall that

$$
\mathrm{d} \bar{u}=-\frac{1}{\alpha} \bar{p} \mathrm{~d}|\bar{u}|
$$

holds. Then we pick any $t_{0}, t_{1} \in \bar{I}$ and estimate using the continuity of $\bar{p}$ in space

$$
\begin{aligned}
\left\|\bar{u}\left(t_{0}\right)-\bar{u}\left(t_{1}\right)\right\|_{\mathcal{M}\left(\Omega_{c}\right)} & =\sup _{\|\phi\|_{\mathcal{C}\left(\Omega_{c}\right)} \leq 1} \int_{\Omega_{c}} \phi(x) \mathrm{d}\left(\bar{u}\left(t_{0}\right)-\bar{u}\left(t_{1}\right)\right) \\
& =\sup _{\|\phi\|_{\mathcal{C}\left(\Omega_{c}\right)} \leq 1} \frac{1}{\alpha} \int_{\Omega_{c}} \phi(x)\left(\bar{p}\left(x, t_{0}\right)-\bar{p}\left(x, t_{1}\right)\right) \mathrm{d}|\bar{u}| \\
& \leq \frac{1}{\alpha}\left\|\bar{p}\left(t_{1}\right)-\bar{p}\left(t_{0}\right)\right\|_{\mathcal{C}\left(\Omega_{c}\right)}\|\bar{u}\|_{\mathcal{M}\left(\Omega_{c}, L^{2}(I)\right)} \\
& \leq c\left|t_{0}-t_{1}\right|^{1 / 2-\varepsilon}\|\bar{u}\|_{\mathcal{M}\left(\Omega_{c}, L^{2}(I)\right)},
\end{aligned}
$$

which implies that

$$
\bar{u} \in \mathcal{C}^{0, \frac{1}{2}-\varepsilon}\left(\bar{I}, \mathcal{M}\left(\Omega_{c}\right)\right) .
$$

Copyright (c) by SIAM. Unauthorized reproduction of this article is prohibited. 
The property $\bar{u} \in \mathcal{C}^{1, \frac{1}{2}-\varepsilon}\left(\bar{I}, \mathcal{M}\left(\Omega_{c}\right)\right)$ in the case $d=1$ can be shown analogously using that $\bar{p} \in \mathcal{C}^{1, \frac{1}{2}-\varepsilon}\left(\bar{I}, \mathcal{C}\left(\Omega_{c}\right)\right)$ and

$$
\mathrm{d} \partial_{t} \bar{u}=-\frac{1}{\alpha} \partial_{t} \bar{p} \mathrm{~d}|\bar{u}|
$$

8. Numerical solution. Next we turn our attention to the discretization of problem $(\mathcal{P})$ by finite elements. For the discretization of the state equation (1.1) we rewrite it as a system of first order equations in time,

$$
\left\{\begin{aligned}
\partial_{t} y-v & =0 & & \text { in } I \times \Omega, \\
\partial_{t} v-\Delta y & =u & & \text { in } I \times \Omega, \\
v=y & =0 & & \text { on } I \times \partial \Omega, \\
(y, v) & =\left(y_{0}, y_{1}\right) & & \text { in }\{0\} \times \Omega .
\end{aligned}\right.
$$

Then the state variables $(y, v)$ are discretized by continuous linear finite elements in time and space. The test functions are chosen from the space of piecewise constant functions in time and piecewise linear, continuous functions in space. Due to the different ansatz and test spaces in time the proposed discretization scheme is a Petrov-Galerkin scheme. The resulting discrete system of equations corresponds to the Crank-Nicolson time stepping scheme applied to the space-discrete version of (8.1). More details on the proposed discretization method for the state equation can be found in [19]. In this paper the authors also derive a gradient-consistent discrete adjoint time stepping scheme. This adjoint scheme corresponds to the discretization of the two adjoint state variables by piecewise constant functions in time and by piecewise linear, continuous functions in space. The discrete control variable has the form

$$
u_{k h}=\sum_{j=1}^{N} u_{j}(t) \delta_{x_{j}}
$$

where the functions $u_{i}(t)$ are piecewise constant and $\delta_{x_{i}}$ are Dirac measures concentrated in the grid points $x_{i}$ of the spatial mesh. Therefore the discrete control cost term has the form

$$
\left\|u_{k h}\right\|_{\mathcal{M}\left(\Omega_{c}, L^{2}(I)\right)}=\sum_{j=1}^{N}\left(\sum_{i=1}^{N_{T}} \tau_{i} u_{j, i}^{2}\right)^{1 / 2},
$$

where $N_{T}$ is the number time steps and $\tau_{i}$ the size of the $i$ th time step. The resulting discrete control cost term is a weighted $l_{1}-l_{2}$-norm in $\mathbb{R}^{N \times N_{T}}$ which is known in compressive sensing in connection with the concept of group sparsity or joint sparsity. This control discretization was also used and analyzed in [20] and [7].

After discretization of all variables problem $(\mathcal{P})$ is a nonsmooth and convex optimization problem in $\mathbb{R}^{N \times N_{T}}$. For the solution of the discrete problem we adapt the strategy of [20]. We add an additional $L^{2}$-regularization term to the cost functional of the continuous problem. The resulting regularized problem is posed in $L^{2}\left(\Omega_{c} \times I\right)$ and the corresponding semismooth Newton method can be applied and analyzed directly on the continuous level (mesh independence). In order to solve the unregularized problem we apply a continuation strategy. The regularization parameter is reduced gradually and the solutions of the regularized subproblems are used for initialization 


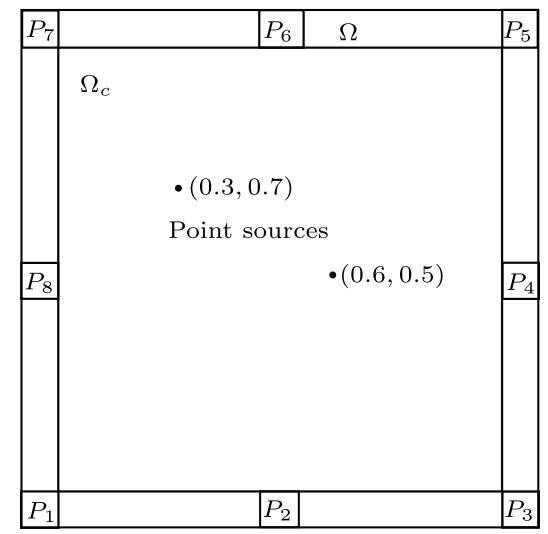

FIG. 9.1. Schematic description of the inverse problem.

of the Newton iterations. This continuation strategy can also be seen as a globalization strategy, since in numerical practice it can be observed that the Newton iteration converges independently of the initial guess only if the regularization parameter is sufficiently large. It can be verified that the solution of the regularized problem converges in the weak-* sense to the solution of the unregularized problem; see [20].

9. Inverse source problem. In this section we use the problem formulation $(\mathcal{P})$ to solve an inverse source problem originating from geophysical sciences. Our problem setup will not fit exactly to the problem formulation $(\mathcal{P})$ and therefore the theoretical findings of the previous sections can be applied only in part.

Seismic events, for example, earthquakes or eruptions of volcanoes, emit seismic waves traveling through the ground. These waves are picked up by seismographs all over the world. Geophysicists use the recorded data to reconstruct the locations and intensities of the initial seismic events. Motivated by such a scenario we intend to solve the following inverse source problem for the acoustic/scalar wave equation (approximation of the elastic wave equation).

Let $\Omega \subset \mathbb{R}^{2}$ be a domain in which $N \geq 1$ sources of the form

$$
u(x, t)=\sum_{i=1}^{N} u_{i}(t) \delta_{x_{i}} \in \mathcal{M}\left(\Omega_{c}, L^{2}(I)\right),
$$

which emit waves. Moreover $M \geq 1$ mean values $z_{j}(t)$ of the emitted waves on patches $P_{j} \subset \Omega$ for $j=1, \ldots, M$ are observed over time. We aim to reconstruct the number of point sources $N$, the locations $x_{i}$, and the time-dependent intensities $u_{i}(t)$ from noisy versions of these seismograms $z_{i}(t)$. The scenario is depicted in Figure 9.1. This inverse problem can be formulated as an optimal control problem of the form

$$
\min _{u, X} \frac{1}{2 M} \sum_{j=1}^{M}\left\|\mathcal{O}_{j}(y)-z_{j}\right\|_{\left.L^{2}(I)\right)}^{2}
$$

Copyright $@$ by SIAM. Unauthorized reproduction of this article is prohibited. 
subject to

$$
\left\{\begin{aligned}
\partial_{t t} y-\Delta y & =\sum_{i=1}^{N} u_{i} \delta_{x_{i}} & & \text { in } I \times \Omega, \\
\partial_{t} y+\partial_{n} y & =0 & & \text { on } I \times \partial \Omega, \\
\left(y, \partial_{t} y\right) & =0 & & \text { in }\{0\} \times \Omega
\end{aligned}\right.
$$

with

$$
\mathcal{O}_{j}(y)(t)=\frac{1}{\left|P_{j}\right|} \int_{P_{j}} y(t, x) \mathrm{d} x, \quad i=1, \ldots, M,
$$

$u=\left\{u_{i}(t)\right\}_{i=1, \ldots, N}, X=\left\{x_{i}\right\}_{i=1, \ldots, N} \subset \Omega_{c}$, and $\partial_{n}$. the normal derivative on $\partial \Omega$. The condition $\partial_{n} y+\partial_{t} y=0$ on $\partial \Omega$ is used as an approximative absorbing boundary condition in order to avoid unrealistic reflections at the boundary; see, e.g., [15].

The optimal control problem (9.2) is not well-posed. If, as a first remedy, the regularization term

$$
u \mapsto \sum_{i=1}^{N}\left\|u_{i}\right\|_{L^{2}(I)}
$$

is added, problem (9.2) is well-posed but not convex since the state $y$ depends nonlinearly on the positions $x_{i}$ of the point sources. In order to obtain a convex problem we instead formulate the optimal control problem in the space $\mathcal{M}\left(\Omega_{c}, L^{2}(I)\right)$ which contains and favors sources of the form (9.1). We therefore suggest casting the inverse problem in the form

$$
\min _{u, y} \frac{1}{2 M} \sum_{j=1}^{M}\left\|\mathcal{O}_{j}(y)-z_{j}\right\|_{L^{2}(I)}^{2}+\alpha\|u\|_{\mathcal{M}\left(\Omega_{c}, L^{2}(I)\right)}
$$

subject to

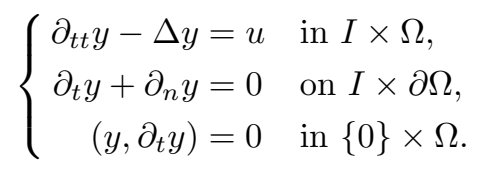

If the optimal control $\bar{u}$ has the form (9.1), it holds that

$$
\|\bar{u}\|_{\mathcal{M}\left(\Omega_{c}, L^{2}(I)\right)}=\sum_{i=1}^{N}\left\|u_{i}\right\|_{L^{2}(I)} .
$$

The problem formulation (9.6) is not covered by our analysis due to the absorbing boundary condition of the state equation and the patchwise observation. The adjoint of the linear observation operator $\mathcal{O}_{j}: L^{2}\left(I, L^{1}(\Omega)\right) \rightarrow L^{2}(I)$ is given by

$$
\mathcal{O}_{j}^{*}: L^{2}(I) \rightarrow L^{2}\left(I, L^{\infty}(\Omega)\right), \quad \mathcal{O}_{j}^{*} q=\frac{1}{\left|P_{j}\right|} \chi_{P_{j}}(x) q(t)
$$

with

$$
\chi_{P_{j}}(x)= \begin{cases}1, & x \in P_{j} \\ 0 & \text { else }\end{cases}
$$

Copyright $@$ by SIAM. Unauthorized reproduction of this article is prohibited. 
and therefore the adjoint state equation has the form

$$
\left\{\begin{aligned}
\partial_{t} \bar{p}-\Delta \bar{p} & =\frac{1}{M} \sum_{j=1}^{M} \mathcal{O}_{j}^{*}\left(\mathcal{O}_{j}(\bar{y})-z_{j}\right) & & \text { in } I \times \Omega, \\
-\partial_{t} \bar{p}+\partial_{n} \bar{p} & =0 & & \text { on } I \times \partial \Omega, \\
\left(\bar{p}, \partial_{t} \bar{p}\right) & =0 & & \text { in }\{T\} \times \Omega .
\end{aligned}\right.
$$

Note that the source term of (9.7) is an element of $L^{2}(I \times \Omega)$. Therefore patchwise observation fits into our framework (cf. Theorem 5.3) in the case of zero Dirichlet boundary conditions. The adjoint waves $\bar{p}$ are triggered by the time reversed misfit terms $\mathcal{O}_{i}-z_{i}$ on the observation patches $P_{i}$ and travel into the domain. The optimal control $\bar{u}$ of problem (9.6) can be represented using the optimal adjoint state $\bar{p}$ (see Proposition 7.2) in the form

$$
\mathrm{d} \bar{u}=-\frac{1}{\alpha} \bar{p} \mathrm{~d}|\bar{u}|
$$

We recall from Proposition 7.2 that

$$
\operatorname{supp}|\bar{u}| \subseteq\left\{x \in \Omega_{c}:\|\bar{p}(x)\|_{L^{2}(I)}=\max _{x \in \Omega_{c}}\|\bar{p}(x)\|_{L^{2}(I)}\right\}
$$

holds. In the case that the function $\|\bar{p}(x)\|_{L^{2}(I)}$ attains its maximum at $\hat{N}$ discrete points in $\Omega_{c}$ the optimal control $\bar{u}$ has the form

$$
\bar{u}(t)=-\frac{1}{\alpha} \sum_{i=1}^{\tilde{N}} c_{i} \bar{p}\left(t, x_{i}\right) \delta_{x_{i}}
$$

with $\tilde{N} \leq \hat{N}$ and constants $c_{i}$. The positions of the maxima of $\|\bar{p}(x)\|_{L^{2}(I)}$ correlate to regions in $\Omega_{c}$ where the adjoint waves overlap. This feature is related to time reversal techniques which are used by geophysicists for the reconstruction of seismic events but are not optimization based; see, e.g., [22].

In our numerical experiment we set $\Omega=(0,1)^{2}, I=[0,1.5], \Omega_{c}=[3 / 16,13 / 16]^{2}$ and the patches $P_{i}$ have the size $8^{-2}$. The time-dependent source functions are chosen as

$$
\begin{aligned}
f_{1}\left(t, t_{s}, \sigma, k\right) & =\frac{1}{\sqrt{2 \pi \sigma}} \sin \left(\frac{k\left(t-t_{s}\right)}{\sigma}\right) e^{-\frac{1}{2}\left(\frac{t-t_{s}}{\sigma}\right)^{2}}, \\
f_{2}\left(t, t_{s}, \sigma\right) & =\frac{2}{\sqrt{3 \sigma \sqrt{\pi}}}\left(1-\left(\frac{t-t_{s}}{\sigma}\right)^{2}\right) e^{-\frac{1}{2}\left(\frac{t-t_{s}}{\sigma}\right)^{2}} .
\end{aligned}
$$

The first is a Gabor wavelet and the second a Ricker wavelet. In our concrete example we intend to reconstruct two point sources and their time-dependent intensities, in particular,

$$
u^{\dagger}(t)=f_{1}(t, 0.3,0.1,2) \delta_{x_{1}}+f_{2}(t, 0.4,0.1) \delta_{x_{2}}
$$

with $x_{1}=(0.3,0.7)$ and $x_{2}=(0.6,0.5)$. The exact intensities and positions are depicted in Figure 9.2. $\quad$ In Figure 9.3 four snapshots of the exact state $y^{\dagger}=S\left(u^{\dagger}\right)$ are shown. The exact state consists of two traveling waves originating from the two point sources. During the evolution of the two waves they interfere with each other. 
$(0.3,0.7)$

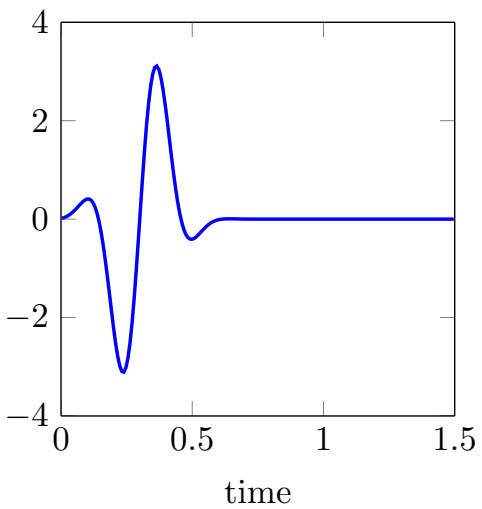

(a) $f_{1}(t, 0.3,0.1,2)$ and $x_{1}$
$(0.6,0.5)$

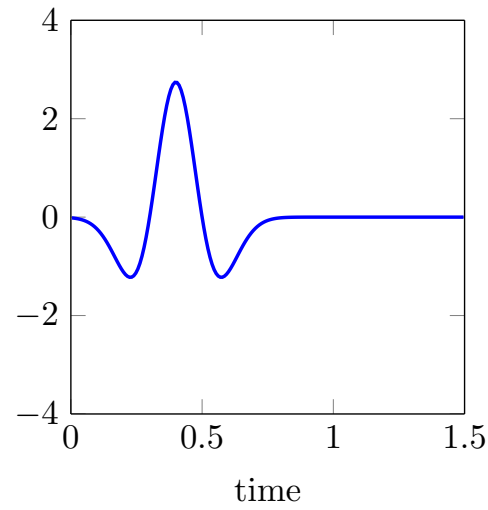

(b) $f_{2}(t, 0.4,0.1)$ and $x_{2}$

FIG. 9.2. Exact intensities and positions.
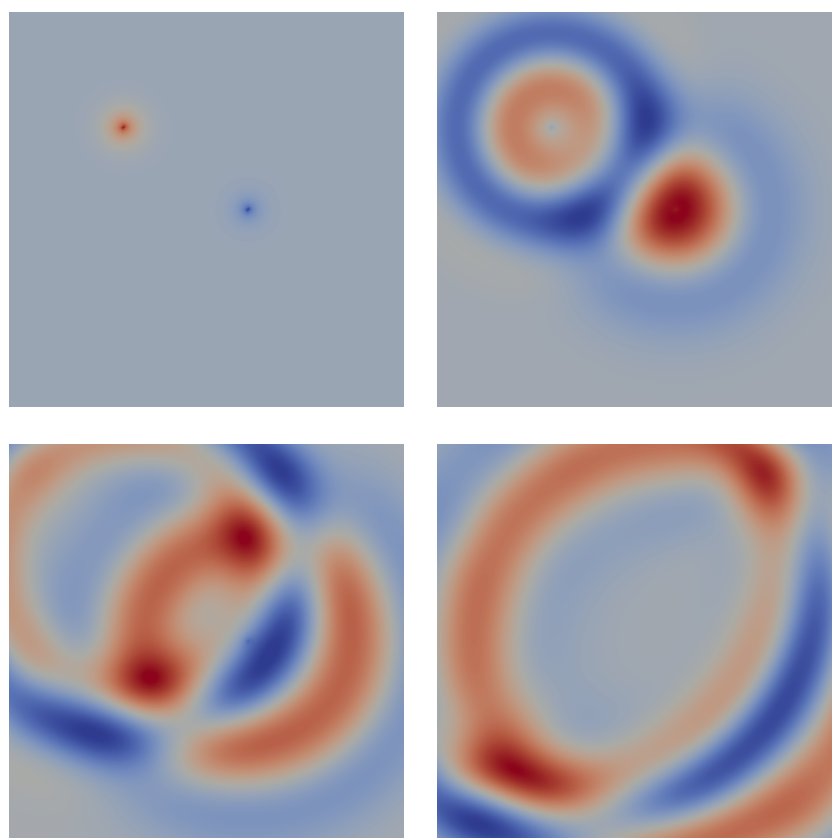

FIG. 9.3. Snapshots of the exact state $y^{\dagger}$ at $t=0.12,0.5,0.7,0.94$.

As can be seen in Figure 9.4 the waves arrive at the observation patches at different times. In this figure we also depict the observations (without noise) and the noisy observations, which are used for the reconstruction. The artificial noisy seismograms are given by $z_{j}=\mathcal{O}_{j}\left(y^{\dagger}\right)+o_{j}$, where, $o_{j} \in L^{2}(I)$ is the background noise. For this example we chose a quite high noise level; in particular, the relative noise level amounts to

$$
\frac{\sum_{j=1}^{M}\left\|o_{j}\right\|_{L^{2}(I)}}{\sum_{j=1}^{M}\left\|\mathcal{O}_{j}\left(y^{\dagger}\right)\right\|_{L^{2}(I)}} \approx 0.4
$$

Copyright $@$ by SIAM. Unauthorized reproduction of this article is prohibited. 


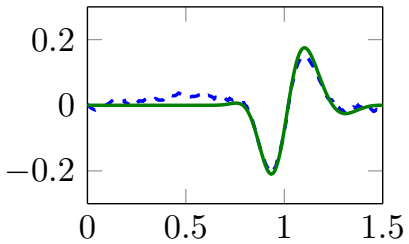

(a) $P_{1}$

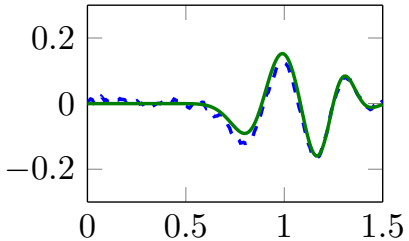

(c) $P_{3}$

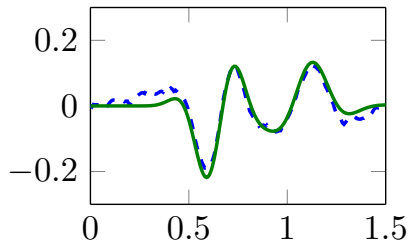

(e) $P_{5}$

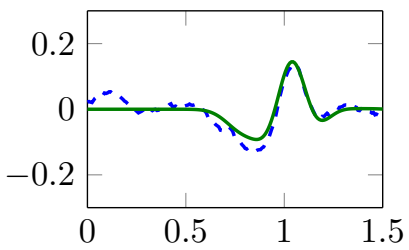

(g) $P_{7}$

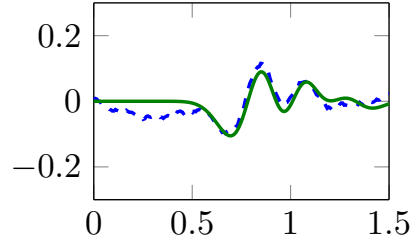

(b) $P_{2}$

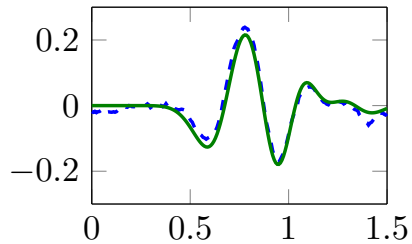

(d) $P_{4}$

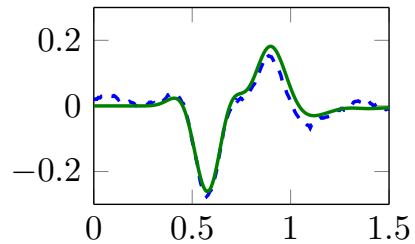

(f) $P_{6}$

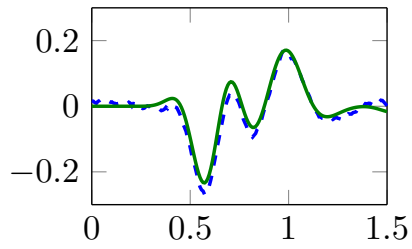

(h) $P_{8}$

FIG. 9.4. Exact and noisy observation (green: exact; blue: noisy).

Figure 9.5 shows the results of the reconstruction process. In Figure 9.5(a) we can see that the total variation measure $|\bar{u}|$ of the reconstruction $\bar{u}$ consists of two point sources which are close to the exact ones. Figure 9.5(b) shows that these positions correlate with the positions where the function $\|\bar{p}(x)\|_{L^{2}(I)}$ attains its maximum on $\Omega_{c}$. The reconstructions of the time-dependent intensities $f_{i}(t)$ are depicted in Figures 9.5(c) and 9.5(d). Their shapes are captured well, but their magnitudes are too small compared to the original ones. This is caused by the structure of our regularization term which regularizes simultaneously in space and time with the same weight $\alpha$. The regularization parameter had to be chosen sufficiently large in order to avoid spurious reconstructions in space caused by the noise. A possible remedy is a postprocessing step consisting of solving problem (9.2) where the positions of the point sources are fixed at the reconstructed values and (9.5) is chosen as the regularization term for the optimization variables $u_{i} \in L^{2}(I)$. In summary, our numerical results give evidence that the proposed formulation produces reconstructions with the desired features. 


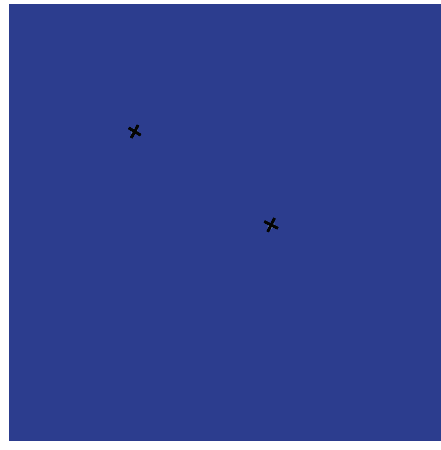

(a) Support of $|\bar{u}|$

$(0.297,0.703)$

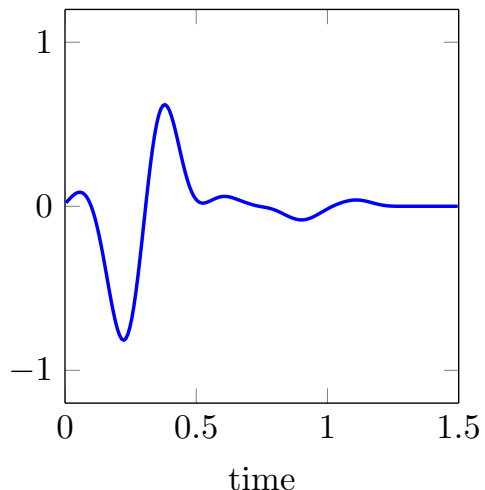

(c) Reconstruction of $f_{1}$ and $x_{1}$

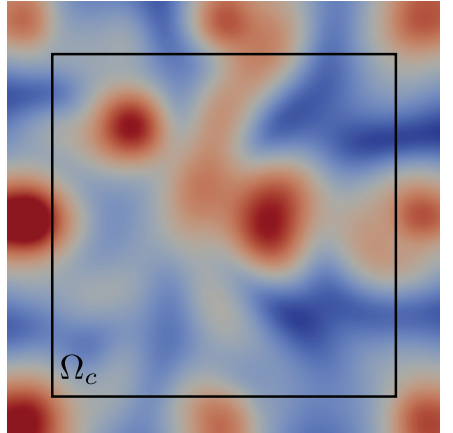

(b) $\|\bar{p}(x)\|_{L^{2}(I)}$ on $\Omega$

$(0.602,0.492)$

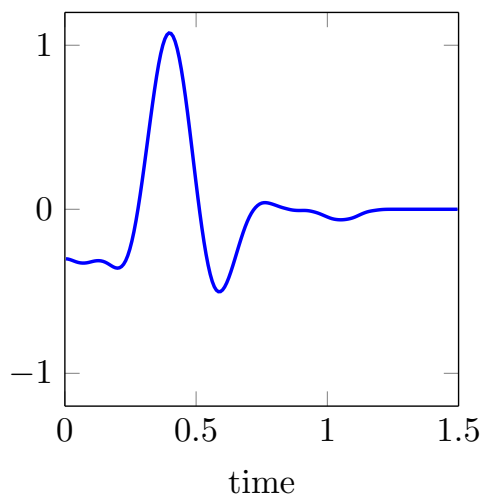

(d) Reconstruction of $f_{2}$ and $x_{2}$

FIG. 9.5. Results of the reconstruction process.

\section{REFERENCES}

[1] H. Amann, Compact embeddings of vector-valued Sobolev and Besov spaces, Glasg. Mat. Ser. III, 35 (2000), pp. 161-177.

[2] A. Bensoussan, G. Da Prato, M. C. Delfour, and S. K. Mitter, Representation and Control of Infinite Dimensional Systems, 2nd ed. Systems Control: Found. Appl., Birkhäuser Boston, Boston, MA, 2007, doi: 10.1007/978-0-8176-4581-6.

[3] V. I. Bogachev, Measure Theory. Vol. I, Springer-Verlag, Berlin, 2007, doi: 10.1007/978-3540-34514-5.

[4] K. Bredies and H. K. Pikkarainen, Inverse problems in spaces of measures, ESAIM Control Optim. Calc. Var., 19 (2013), pp. 190-218, doi: 10.1051/cocv/2011205.

[5] H. BRÉZIS, Opérateurs maximaux monotones et semi-groupes de contractions dans les espaces de Hilbert, North-Holland Math. Stud. 5, North-Holland, Amsterdam, 1973.

[6] H. Brezis, Functional Analysis, Sobolev Spaces and Partial Differential Equations, Universitext, Springer, New York, 2011.

[7] E. Casas, C. Clason, and K. Kunisch, Parabolic control problems in measure spaces with sparse solutions, SIAM J. Control Optim., 51 (2013), pp. 28-63, doi: 10.1137/120872395.

[8] E. CaSAS AND K. Kunisch, Optimal control of semilinear elliptic equations in measure spaces, SIAM J. Control Optim., 52 (2014), pp. 339-364, doi: 10.1137/13092188X.

[9] E. Casas, B. Vexler, ANd E. Zuazua, Sparse initial data indentification for parabolic PDE and its finite element approximations, Math. Control Relat. Fields, 5 (2015), pp. 377-399, doi: 10.3934/mcrf.2015.5.377.

[10] E. Casas and E. Zuazua, Spike controls for elliptic and parabolic PDEs, Systems Control Lett., 62 (2013), pp. 311-318, doi: 10.1016/j.sysconle.2013.01.001. 
[11] C. Clason and K. Kunisch, A duality-based approach to elliptic control problems in nonreflexive Banach spaces, ESAIM Control Optim. Calc. Var., 17 (2011), pp. 243-266, doi: $10.1051 / \mathrm{cocv} / 2010003$.

[12] C. Clason and K. Kunisch, A measure space approach to optimal source placement, Comput. Optim. Appl., 53 (2011), pp. 155-171.

[13] S. S. Dragomin, Some Gronwall Type Inequalities and Applications, Nova Science Publishers, Hauppauge, NY, 2003.

[14] I. Ekeland And R. TÉmam, Convex Analysis and Variational Problems, Classics in Appl. Math., SIAM, Philadelphia, PA, english ed., 1999, doi: 10.1137/1.9781611971088.

[15] B. EngQuist AND A. MAJDA, Absorbing boundary conditions for numerical simulation of waves, Proc. Natl. Acad. Sci. USA, 74 (1977), pp. 1765-1766.

[16] M. FornAsier AND H. RAUHUT, Recovery algorithms for vector-valued data with joint sparsity constraints, SIAM J. Numer. Anal., 46 (2008), pp. 577-613, doi: 10.1137/0606668909.

[17] W. Hensgen, A simple proof of Singer's representation theorem, Proc. Amer. Math. Soc., 124 (1996), pp. 3211-3212, doi: 10.1090/S0002-9939-96-03493-4.

[18] R. Herzog, G. Stadler, and G. Wachsmuth, Directional sparsity in optimal control of partial differential equations, SIAM J. Control Optim., 50 (2012), pp. 943-963, doi: $10.1137 / 100815037$.

[19] A. Kröner, K. Kunisch, And B. Vexler, Semismooth Newton methods for optimal control of the wave equation with control constraints, SIAM J. Control Optim., 49 (2011), pp. 830858, doi: 10.1137/090766541.

[20] K. Kunisch, K. Pieper, And B. Vexler, Measure valued directional sparsity for parabolic optimal control problems, SIAM J. Control Optim., 52 (2014), pp. 3078-3108.

[21] S. LANG, Real Analysis, 2nd ed., Addison-Wesley, Reading, MA, 1983.

[22] C. Larmat, J.-P. Montagner, M. Fink, Y. Capdeville, A. Tourin, and E. Clevede, Time-reversal imaging of seismic sources and application to the great sumatra earthquake, Geophys. Res. Lett., 33 (2006), doi: 10.1029/2006GL026336.

[23] I. Lasiecka, J.-L. Lions, and R. Triggiani, Non homogeneous boundary value problems for second order hyperbolic operators, J. Math. Pures Appl., 65 (1986), pp. 149-192.

[24] I. Lasiecka and R. Triggiani, Control Theory for Partial Differential Equations: Continuous and Approximation Theories. II, Encyclopedia Math. Appl., Cambridge University Press, Cambridge, UK, 2000, doi: 10.1017/CBO9780511574801.002

[25] J. L. Lions, Pointwise control for distributed systems, in Control and Estimation in distributed parameter systems, Frontiers in Appl. Math. 11, SIAM, Philadelphia, 1992, pp. 1-39, doi: 10.1137/1.9781611970982.ch1.

[26] J.-L. Lions and E. Magenes, Non-homogeneous Boundary Value Problems and Applications, Vol. I, Grundlehren Math. Wiss. 181, Springer-Verlag, New York, 1972.

[27] R. E. Showalter, Monotone Operators in Banach Space and Nonlinear Partial Differential Equations, Math. Surveys Monogr., AMS, Providence, RI, 1997.

[28] R. Triggiani, Regularity with interior point control. I. Wave and Euler-Bernoulli equations, in Boundary Control and Boundary Variation, Lecture Notes in Control and Inform. Sci., 178, Springer, Berlin, 1992, pp. 321-355, doi: 10.1007/BFb0006705.

Copyright (C) by SIAM. Unauthorized reproduction of this article is prohibited. 